\title{
ASYMPTOTICS OF ALMOST HOLOMORPHIC SECTIONS OF AMPLE LINE BUNDLES ON SYMPLECTIC MANIFOLDS
}

\author{
BERNARD SHIFFMAN AND STEVE ZELDITCH
}

\begin{abstract}
In their work on symplectic manifolds, Donaldson and Auroux use analogues of holomorphic sections of an ample line bundle $L$ over a symplectic manifold $M$ to create symplectically embedded zero sections and almost holomorphic maps to various spaces. Their analogues were termed 'asymptotically holomorphic' sequences $\left\{s_{N}\right\}$ of sections of $L^{N}$. We study another analogue $H_{J}^{0}\left(M, L^{N}\right)$ of holomorphic sections, which we call 'almostholomorphic' sections, following a method introduced earlier by Boutet de Monvel - Guillemin [BoGu in a general setting of symplectic cones. By definition, sections in $H_{J}^{0}\left(M, L^{N}\right)$ lie in the range of a Szegö projector $\Pi_{N}$. Starting almost from scratch, and only using almostcomplex geometry, we construct a simple parametrix for $\Pi_{N}$ of precisely the same type as the Boutet de Monvel-Sjöstrand parametrix in the holomorphic case [BoSj]. We then show that $\Pi_{N}(x, y)$ has precisely the same scaling asymptotics as does the holomorphic Szegö kernel as analyzed in BSZ1. The scaling asymptotics imply more or less immediately a number of analogues of well-known results in the holomorphic case, e.g. a Kodaira embedding theorem and a Tian almost-isometry theorem. We also explain how to modify Donaldson's constructions to prove existence of quantitatively transverse sections in $H_{J}^{0}\left(M, L^{N}\right)$.
\end{abstract}

\section{Contents}

Introduction 2

1. Circle bundles and almost CR geometry 4

1.1. Almost complex symplectic manifolds 4

1.2. The circle bundle and Heisenberg coordinates 5

2. Almost holomorphic sections and Szegö kernels 8

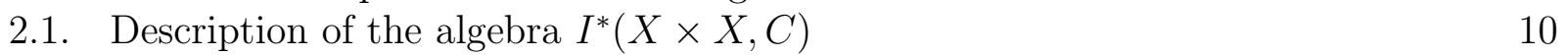

$\begin{array}{lll}2.2 . & \text { The positive Lagrangean ideal } & 11\end{array}$

\begin{tabular}{|lll}
\hline 2.3. & The complex canonical relation & 14
\end{tabular}

$\begin{array}{lll}2.4 . & \text { The Szegö projector } & 19\end{array}$

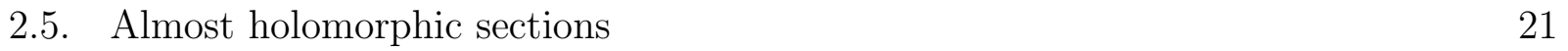

3. Scaling asymptotics for Szegö kernels 22

4. Kodaira embedding and Tian almost isometry theorem 28

$\begin{array}{lll}5 . & \text { Transversal sections } & 30\end{array}$

\begin{tabular}{lll}
\hline 5.1. & Quantitative transversality & 30
\end{tabular}

5.2. The Adjunction Formula 34

\begin{tabular}{ll}
\hline References & 36
\end{tabular}

Date: March 27, 2001.

Research partially supported by NSF grants DMS-9800479 (first author) and DMS-9703775, DMS-0071358 (second author). 


\section{INTRODUCTION}

This paper is concerned with asymptotically holomorphic sections of ample line bundles over almost-complex symplectic manifolds $(M, J, \omega)$. Such line bundles and sections are symplectic analogues of the usual objects in complex algebraic geometry. Interest in their properties has grown in recent years because of their use by Donaldson [Don1, Don2], Auroux Aur1, Aur2 and others [AuKa, Sik in proving symplectic analogues of standard results in complex geometry. These results involve properties of asymptotically holomorphic sections of high powers of the bundle, particularly those involving their zero sets and the maps they define to projective space.

We take up the study of asymptotically holomorphic sections from the viewpoint of the microlocal analysis of the $\bar{\partial}$ operator on a symplectic almost-complex manifold, and define a class of 'almost holomorphic sections' by a method due to Boutet de Monvel and Guillemin Bout, BoGu. Sections $s$ of powers of a complex line bundle $L^{N} \rightarrow M$ over $M$ are identified with equivariant functions $\hat{s}$ on the associated $S^{1}$-bundle $X$, and the $\bar{\partial}$ operator on sections is identified with the $\bar{\partial}_{b}$ operator on $X$. In the non-integrable almost-complex symplectic case there are in general no solutions of $\bar{\partial}_{b} s=0$. To define an 'almost holomorphic section' $s$, Boutet de Monvel and Guillemin define a certain (pseudodifferential) $\bar{D}$-complex over $X$ [BoGu BoSj]. The space $H_{J}^{0}\left(M, L^{N}\right)$ of almost holomorphic sections is then the space of sections corresponding to solutions of $\bar{D}_{0} s=0$. The operator $\bar{D}_{0}$ is not uniquely or even canonically defined, and it is difficult to explicitly write down these almost holomorphic sections. However, our main results show that on small length scales (the same ones used in [Don1]), these almost holomorphic sections have the same asymptotic behavior as holomorphic sections in the $N \rightarrow \infty$ limit. Furthermore, as we shall show in a related paper [ShZe2], typical sections have globally similar properties to the asymptotically holomorphic sections of Donaldson and Auroux.

The fundamental ingredient of these statements is the scaling asymptotics of the almostholomorphic Szegö kernels $\Pi_{N}(z, w)$, i.e. the orthogonal projections onto $H_{J}^{0}\left(M, L^{N}\right)$. As our results will show, the 'peak sections' $z \mapsto \Pi_{N}(z, w)$ about points $w \in M$ are (up to normalization) intrinsically defined almost holomorphic sections with the main properties of Donaldson's exponentially localized sections (cf. Don1, Proposition 11). Analytically, it is more convenient to work with the lift of $\Pi_{N}$ as an equivariant kernel $\Pi_{N}(x, y)$ on $X \times X$ than as a section of $L^{N} \otimes L^{* N}$, so henceforth we will use the term Szegö kernel for the equivariant kernel.

The scaling asymptotics of $\Pi_{N}(x, y)$ must be given in special 'Heisenberg' coordinates on $X$, defined as follows: Choose 'normal' local coordinates $\left\{z_{j}\right\}$ centered at a point $P_{0} \in M$ as in Section 1.1 and choose a 'preferred' local frame for $L$, which together with the coordinates on $M$ give us 'Heisenberg coordinates' $x=(z, \theta), y=(w, \varphi)$ on $X$ (see $\S 1.2$ ). We obtain the following near-diagonal asymptotic formula for the Szegö kernel in terms of these Heisenberg coordinates:

THEOREM 1. We have:

$$
\begin{aligned}
& N^{-m} \prod_{N}\left(P_{0}+\frac{u}{\sqrt{N}}, \frac{\theta}{N} ; P_{0}+\frac{v}{\sqrt{N}}, \frac{\varphi}{N}\right) \\
& =\frac{1}{\pi^{m}} e^{i(\theta-\varphi)+u \cdot \bar{v}-\frac{1}{2}\left(|u|^{2}+|v|^{2}\right)}\left[1+\sum_{r=1}^{K} N^{-\frac{r}{2}} b_{r}\left(P_{0}, u, v\right)+N^{-\frac{K+1}{2}} R_{K}\left(P_{0}, u, v, N\right)\right], \\
& \quad \text { where }\left\|R_{K}\left(P_{0}, u, v, N\right)\right\|_{\mathcal{C}^{j}(\{|u|+|v| \leq \rho\})} \leq C_{K, j, \rho} \text { for } j=1,2,3, \ldots .
\end{aligned}
$$


A more precise statement will be given in Theorem 3.1. The proof of Theorem 1 is based on the construction of explicit parametrices for $\Pi$ and its Fourier coefficients $\Pi_{N}$. These parametrices closely resemble those of Boutet de Monvel - Sjöstrand [BoSj] in the holomorphic case. The construction is new but closely follows the work of Menikoff and Sjöstrand [MenSj], Sjö] and of Boutet de Monvel and Guillemin [Bout, BoGu]. For the sake of concreteness, we will give a fairly detailed exposition of the construction of the zeroth term of the $\bar{D}_{j}$ complex and of the Szegö kernel.

As more or less immediate corollaries of the scaling asymptotics, we prove the following symplectic analogues of the holomorphic Kodaira embedding theorem and Tian almostisometry theorem [Tian]. These concern the analogue of the usual Kodaira map $\Phi_{N}: M \rightarrow$ $\mathbb{C P}^{d_{N}-1}$ determined by the space $H_{J}^{0}\left(M, L^{N}\right)$.

TheOREM 2. Let $L \rightarrow(M, \omega)$ be the pre-quantum line bundle over a $2 m$-dimensional symplectic manifold, and let $\left\{\Phi_{N}\right\}$ be its Kodaira maps. Then:

(a) There exists a complete asymptotic expansion:

$$
\Pi_{N}(z, 0 ; z, 0)=a_{0} N^{m}+a_{1}(z) N^{m-1}+a_{2}(z) N^{m-2}+\ldots
$$

for certain smooth coefficients $a_{j}(z)$ with $a_{0}=\pi^{-m}$. Hence, the maps $\Phi_{N}$ are well-defined for $N \gg 0$.

(b) Let $\omega_{F S}$ denote the Fubini-Study form on $\mathbb{C P}^{d_{N}-1}$. Then

$$
\left\|\frac{1}{N} \Phi_{N}^{*}\left(\omega_{F S}\right)-\omega\right\|_{\mathcal{C}^{k}}=O\left(\frac{1}{N}\right)
$$

for any $k$.

Theorem 3. The Kodaira map $\Phi_{N}: M \rightarrow \mathbb{C P}^{d_{N}-1}$ is an embedding, for $N$ sufficiently large.

We should draw attention to related work of Borthwick-Uribe [BoUr1, BoUr2], which defines 'almost holomorphic' in a somewhat different way, and proves analogues of the Tian almost-isometry and Kodaira embedding theorems in their setting. The Borthwick-Uribe proof of the almost-complex Tian theorem was in turn motivated by a similar proof in the holomorphic case in Ze]. The approach we take here, based on the off-diagonal scaling asymptotics of the Szegö kernel, is new and we believe it will have further geometric applications.

Finally, we use the scaling asymptotics of the Szegö kernel $\Pi_{N}(x, y)$ to show that Donaldson's symplectic embedding theorem and the genus calculation of embedded symplectic curves can be adapted to the setting of almost holomorphic sections. We will see in $\S 5$ that the proof in [Don1] of the existence of quantitatively transversal asymptotically holomorphic sections can be adapted to prove the existence of quantitatively transverse sections in $H_{J}^{0}\left(M, L^{N}\right)$.

Further applications of the scaling asymptotics in Theorems 1 and 3.1 will be given in a forthcoming paper [ShZe2], in which we discuss typical properties of almost holomorphic sections (in the probabilistic sense of BBSZ1, BSZ2]). In particular, we show that almost every sequence of $\mathcal{L}^{2}$-normalized sections $\left\{s_{N}\right\} \in S H_{J}^{0}\left(M, L^{N}\right)$ satisfies the estimates:

$$
\begin{array}{ll}
\left\|s_{N}\right\|_{\infty}=O(\sqrt{\log N}), \quad\left\|\nabla^{k} s_{N}\right\|_{\infty}=O\left(N^{k / 2} \sqrt{\log N}\right), \\
\left\|\bar{\partial} s_{N}\right\|_{\infty}=O(\sqrt{\log N}), \quad\left\|\nabla^{k} \bar{\partial} s_{N}\right\|_{\infty}=O\left(N^{k / 2} \sqrt{\log N}\right), \quad(k \geq 1) .
\end{array}
$$


Consequently, the sequence of almost-holomorphic sections $\left\{\frac{1}{\sqrt{\log N}} s_{N}\right\}$ is almost surely asymptotically holomorphic in the sense of Donaldson and Auroux Don1, Aur1.

We also use Theorem 3.1 to show, in joint work with P. Bleher [B] [BSZ1, Theorem 3.6] on the universality of scaling limits of correlations of zeros of sections of positive line bundles over complex manifolds extends to almost holomorphic sections on symplectic manifolds. Thus, on small balls (of radius $1 / \sqrt{N}$ ), almost holomorphic sections have the same asymptotic probabilities as holomorphic sections of taking on specified values and derivatives.

For the readers' convenience, we conclude this introduction with a brief outline of the paper. We begin in $\S 1$ by describing some terminology from symplectic geometry and introducing our Heisenberg coordinates. In \$2, we use the method of Boutet de Monvel, Guillemin, and Sjöstrand [Bout, BoGu, BoSj] to construct Szegö projectors $\Pi_{N}$, which we show are complex Fourier integral operators of the same type as in the holomorphic case, and then to provide a Boutet de Monvel-Guillemin complex of pseudodifferential operators, which replaces the $\bar{\partial}_{b}$ complex in the symplectic setting. The zeroth term of this complex is used to define sequences of almost holomorphic sections analogous to the integrable complex case $(\$ 2.5)$. In $\S 3$, we obtain the scaling asymptotics of $\Pi_{N}\left(P_{0}+\frac{u}{\sqrt{N}}, \frac{\theta}{N} ; P_{0}+\frac{v}{\sqrt{N}}, \frac{\varphi}{N}\right)$, which we apply in $\S$ to prove Theorems 2 and 3 on the symplectic Kodaira map. Finally, in $\S 5$, we show how concentrated almost holomorphic sections can be used in place of asymptotically holomorphic sections to construct quantitatively transversal sections by Donaldson's method.

We would like to thank Michael Christ for informative comments concerning the offdiagonal decay of the Szegö kernel in the holomorphic case.

\section{Circle Bundles And Almost CR geometry}

We denote by $(M, \omega)$ a compact symplectic manifold such that $\left[\frac{1}{\pi} \omega\right]$ is an integral cohomology class. As is well known (cf. Wod, Prop. 8.3.1]; see also [GuSt), there exists a hermitian line bundle $(L, h) \rightarrow M$ and a metric connection $\nabla$ on $L$ whose curvature $\Theta_{L}$ satisfies $\frac{i}{2} \Theta_{L}=\omega$. We denote by $L^{N}$ the $N^{\text {th }}$ tensor power of $L$. The 'quantization' of $(M, \omega)$ at Planck constant $1 / N$ should be a Hilbert space of polarized sections of $L^{N}$ ( GuSt, p. 266]). In the complex case, polarized sections are simply holomorphic sections. The notion of polarized sections is problematic in the non-complex symplectic setting, since the Lagrangean subbundle $T^{1,0} M$ defining the complex polarization is not integrable and there usually are no 'holomorphic' sections. A subtle but compelling replacement for the notion of polarized section has been proposed by Boutet de Monvel and Guillemin [Bout, BoGu], and it is this notion which we adopt in this paper. For the asymptotic analysis, it is best to view sections of $L^{N}$ as functions on the unit circle bundle $X \subset L^{*}$; we shall describe the 'almost CR geometry' of $X$ in $\$ 1.2$ below.

1.1. Almost complex symplectic manifolds. We begin by reviewing some terminology from almost complex symplectic geometry. An almost complex symplectic manifold is a symplectic manifold $(M, \omega)$ together with an almost complex structure $J$ satisfying the compatibility condition $\omega(J v, J w)=\omega(v, w)$ and the positivity condition. $\omega(v, J v)>0$. We give $M$ the Riemannian metric $g(v, w)=\omega(v, J w)$. We denote by $T^{1,0} M$, resp. $T^{0,1} M$, the holomorphic, resp. anti-holomorphic, sub-bundle of the complex tangent bundle $T M$; i.e., 
$J=i$ on $T^{1,0} M$ and $J=-i$ on $T^{0,1} M$. We give $M$ local coordinates $\left(x_{1}, y_{1}, \ldots, x_{m}, y_{m}\right)$, and we write $z_{j}=x_{j}+i y_{j}$. As in the integrable (i.e., holomorphic) case, we let $\left\{\frac{\partial}{\partial z_{j}}, \frac{\partial}{\partial \bar{z}_{j}}\right\}$ denote the dual frame to $\left\{d z_{j}, d \bar{z}_{j}\right\}$. Although in our case, the coordinates $z_{j}$ are not holomorphic and consequently $\frac{\partial}{\partial z_{j}}$ is generally not in $T^{1,0} M$, we nonetheless have

$$
\frac{\partial}{\partial z_{j}}=\frac{1}{2} \frac{\partial}{\partial x_{j}}-\frac{i}{2} \frac{\partial}{\partial y_{j}}, \quad \frac{\partial}{\partial \bar{z}_{j}}=\frac{1}{2} \frac{\partial}{\partial x_{j}}+\frac{i}{2} \frac{\partial}{\partial y_{j}} .
$$

At any point $P_{0} \in M$, we can choose a local frame $\left\{\bar{Z}_{1}^{M}, \ldots, \bar{Z}_{m}^{M}\right\}$ for $T^{0,1} M$ near $P_{0}$ and coordinates about $P_{0}$ so that

$$
\bar{Z}_{j}^{M}=\frac{\partial}{\partial \bar{z}_{j}}+\sum_{k=1}^{m} B_{j k}(z) \frac{\partial}{\partial z_{k}}, \quad B_{j k}\left(P_{0}\right)=0,
$$

and hence $\partial /\left.\partial z_{j}\right|_{P_{0}} \in T^{1,0}(M)$. This is one of the properties of our 'preferred coordinates' defined below.

Definition: Let $P_{0} \in M$. A coordinate system $\left(z_{1}, \ldots, z_{m}\right)$ on a neighborhood $U$ of $P_{0}$ is preferred at $P_{0}$ if

$$
\sum_{j=1}^{m} d z_{j} \otimes d \bar{z}_{j}=\left.(g-i \omega)\right|_{P_{0}}
$$

In fact, the coordinates $\left(z_{1}, \ldots, z_{m}\right)$ are preferred at $P_{0}$ if an only if any two of the following conditions (and hence all three) are satisfied:

i) $\partial /\left.\partial z_{j}\right|_{P_{0}} \in T^{1,0}(M)$, for $1 \leq j \leq m$,

ii) $\omega\left(P_{0}\right)=\omega_{0}$,

iii) $g\left(P_{0}\right)=g_{0}$

where $\omega_{0}$ is the standard symplectic form and $g_{0}$ is the Euclidean metric:

$$
\omega_{0}=\frac{i}{2} \sum_{j=1}^{m} d z_{j} \wedge d \bar{z}_{j}=\sum_{j=1}^{m}\left(d x_{j} \otimes d y_{j}-d y_{j} \otimes d x_{j}\right), \quad g_{0}=\sum_{j=1}^{m}\left(d x_{j} \otimes d x_{j}+d y_{j} \otimes d y_{j}\right) .
$$

(To verify this statement, note that condition (i) is equivalent to $J\left(d x_{j}\right)=-d y_{j}$ at $P_{0}$, and use $g(v, w)=\omega(v, J w)$.) Note that by the Darboux theorem, we can choose the coordinates so that condition (ii) is satisfied on a neighborhood of $P_{0}$, but this is not necessary for our scaling results.

1.2. The circle bundle and Heisenberg coordinates. We now let $(M, \omega, J)$ be a compact, almost complex symplectic manifold such that $\left[\frac{1}{\pi} \omega\right]$ is an integral cohomology class, and we choose a hermitian line bundle $(L, h) \rightarrow M$ and a metric connection $\nabla$ on $L$ with $\frac{i}{2} \Theta_{L}=\omega$. In order to simultaneously analyze sections of all positive powers $L^{N}$ of the line bundle $L$, we work on the associated principal $S^{1}$ bundle $X \rightarrow M$, which is defined as follows: let $\pi: L^{*} \rightarrow M$ denote the dual line bundle to $L$ with dual metric $h^{*}$, and put $X=\left\{v \in L^{*}:\|v\|_{h^{*}}=1\right\}$. We let $\alpha$ be the the connection 1 -form on $X$ given by $\nabla$; we then have $d \alpha=\pi^{*} \omega$, and thus $\alpha$ is a contact form on $X$, i.e., $\alpha \wedge(d \alpha)^{m}$ is a volume form on $X$.

We let $r_{\theta} x=e^{i \theta} x(x \in X)$ denote the $S^{1}$ action on $X$ and denote its infinitesimal generator by $\frac{\partial}{\partial \theta}$. A section $s$ of $L$ determines an equivariant function $\hat{s}$ on $L^{*}$ by the rule $\hat{s}(\lambda)=(\lambda, s(z))$ $\left(\lambda \in L_{z}^{*}, z \in M\right)$. It is clear that if $\tau \in \mathbb{C}$ then $\hat{s}(z, \tau \lambda)=\tau \hat{s}$. We henceforth restrict $\hat{s}$ to $X$ 
and then the equivariance property takes the form $\hat{s}\left(r_{\theta} x\right)=e^{i \theta} \hat{s}(x)$. Similarly, a section $s_{N}$ of $L^{N}$ determines an equivariant function $\hat{s}_{N}$ on $X$ : put

$$
\hat{s}_{N}(\lambda)=\left(\lambda^{\otimes N}, s_{N}(z)\right), \quad \lambda \in X_{z},
$$

where $\lambda^{\otimes N}=\lambda \otimes \cdots \otimes \lambda$; then $\hat{s}_{N}\left(r_{\theta} x\right)=e^{i N \theta} \hat{s}_{N}(x)$. We denote by $\mathcal{L}_{N}^{2}(X)$ the space of such equivariant functions transforming by the $N^{\text {th }}$ character.

In the complex case, $X$ is a $\mathrm{CR}$ manifold. In the general almost-complex symplectic case it is an almost CR manifold. The almost $C R$ structure is defined as follows: The kernel of $\alpha$ defines a horizontal hyperplane bundle $H \subset T X$. Using the projection $\pi: X \rightarrow M$, we may lift the splitting $T M=T^{1,0} M \oplus T^{0,1} M$ to a splitting $H=H^{1,0} \oplus H^{0,1}$. The almost CR structure on $X$ is defined to be the splitting $T X=H^{1,0} \oplus H^{0,1} \oplus \mathbb{C} \frac{\partial}{\partial \theta}$. We also consider a local orthonormal frame $Z_{1}, \ldots, Z_{n}$ of $H^{1,0}$, resp. $\bar{Z}_{1}, \ldots, \bar{Z}_{m}$ of $H^{0,1}$, and dual orthonormal coframes $\vartheta_{1}, \ldots, \vartheta_{m}$, resp. $\bar{\vartheta}_{1}, \ldots, \bar{\vartheta}_{m}$. On the manifold $X$ we have $d=\partial_{b}+\bar{\partial}_{b}+\frac{\partial}{\partial \theta} \otimes \alpha$, where $\partial_{b}=\sum_{j=1}^{m} \vartheta_{j} \otimes Z_{j}$ and $\bar{\partial}_{b}=\sum_{j=1}^{m} \bar{\vartheta}_{j} \otimes \bar{Z}_{j}$. We define the almost-CR $\bar{\partial}_{b}$ operator by $\bar{\partial}_{b}=\left.d f\right|_{H^{1,0}}$. Note that for an $\mathcal{L}^{2}$ section $s^{N}$ of $L^{N}$, we have

$$
\left(\nabla_{L^{N}} s^{N}\right)^{\widehat{-}}=d^{h} \hat{s}^{N}
$$

where $d^{h}=\partial_{b}+\bar{\partial}_{b}$ is the horizontal derivative on $X$.

Our near-diagonal asymptotics of the Szegö kernel (§3) are given in terms of the Heisenberg dilations, using local 'Heisenberg coordinates' at a point $x_{0} \in X$. To describe these coordinates, we first need the concept of a 'preferred frame':

Definition: A preferred frame for $L \rightarrow M$ at a point $P_{0} \in M$ is a local frame $e_{L}$ in a neighborhood of $P_{0}$ such that

$$
\begin{aligned}
\text { i) } & \left\|e_{L}\right\|_{P_{0}}=1 ; \\
\text { ii) } & \left.\nabla e_{L}\right|_{P_{0}}=0 ; \\
\text { iii) } & \left.\nabla^{2} e_{L}\right|_{P_{0}}=-\left.(g+i \omega) \otimes e_{L}\right|_{P_{0}} \in T_{M}^{*} \otimes T_{M}^{*} \otimes L .
\end{aligned}
$$

(A preferred frame can be constructed by multiplying an arbitrary frame by a function with specified 2-jet at $P_{0}$; any two such frames agree to third order at $P_{0}$.) Once we have property (ii), property (iii) is independent of the choice of connection on $T_{M}^{*}$ used to define $\nabla: \mathcal{C}^{\infty}\left(M, L \otimes T_{M}^{*}\right) \rightarrow \mathcal{C}^{\infty}\left(M, L \otimes T_{M}^{*} \otimes T_{M}^{*}\right)$. In fact, property (iii) is a necessary condition for obtaining universal scaling asymptotics, because of the 'parabolic' scaling in the Heisenberg group. Note that if $e_{L}$ is a preferred frame at $P_{0}$ and if $\left(z_{1}, \ldots, z_{m}\right)$ are preferred coordinates at $P_{0}$, then we compute the Hessian of $\left\|e_{L}\right\|$ :

$$
\left(\nabla^{2}\left\|e_{L}\right\|_{h}\right)_{P_{0}}=\Re\left(\nabla^{2} e_{L}, e_{L}\right)_{P_{0}}=-g\left(P_{0}\right) ;
$$

thus if the preferred coordinates are 'centered' at $P_{0}$ (i.e., $P_{0}=0$ ), we have

$$
\left\|e_{L}\right\|_{h}=1-\frac{1}{2}|z|^{2}+O\left(|z|^{3}\right) \text {. }
$$

Remark: Recall ( BSZ1, §1.3.2]) that the Bargmann-Fock representation of the Heisenberg group acts on the space of holomorphic functions on $(M, \omega)=\left(\mathbb{C}^{m}, \omega_{0}\right)$ that are square integrable with respect to the weight $h=e^{-\left|z^{2}\right|}$. We let $L=\mathbb{C}^{m} \times \mathbb{C}$ be the trivial bundle. 
Then the trivializing section $e_{L}(z):=(z, 1)$ is a preferred frame at $P_{0}=0$ with respect to the Hermitian connection $\nabla$ given by

$$
\nabla e_{L}=\partial \log h \otimes e_{L}=-\sum_{j=1}^{m} \bar{z}_{j} d z_{j} \otimes e_{L} .
$$

Indeed, the above yields $\left.\nabla^{2} e_{L}\right|_{0}=-\sum d \bar{z}_{j} \otimes d z_{j} \otimes e_{L}(0)=-\left(g_{0}+i \omega_{0}\right) \otimes e_{L}(0)$.

The preferred frame and preferred coordinates together give us 'Heisenberg coordinates':

Definition: A Heisenberg coordinate chart at a point $x_{0}$ in the principal bundle $X$ is a coordinate chart $\rho: U \approx V$ with $0 \in U \subset \mathbb{C}^{m} \times \mathbb{R}$ and $\rho(0)=x_{0} \in V \subset X$ of the form

$$
\rho\left(z_{1}, \ldots, z_{m}, \theta\right)=e^{i \theta} a(z)^{-\frac{1}{2}} e_{L}^{*}(z),
$$

where $e_{L}$ is a preferred local frame for $L \rightarrow M$ at $P_{0}=\pi\left(x_{0}\right)$, and $\left(z_{1}, \ldots, z_{m}\right)$ are preferred coordinates centered at $P_{0}$. (Note that $P_{0}$ has coordinates $(0, \ldots, 0)$ and $e_{L}^{*}\left(P_{0}\right)=x_{0}$.)

We now give some computations using local coordinates $\left(z_{1}, \ldots, z_{m}, \theta\right)$ of the form (5) for a local frame $e_{L}$. (For the moment, we do not assume they are Heisenberg coordinates.) We write

$$
\begin{aligned}
a(z) & =\left\|e_{L}^{*}(z)\right\|_{h^{*}}^{2}=\left\|e_{L}(z)\right\|_{h}^{-2}, \\
\alpha & =d \theta+\beta, \quad \beta=\sum_{j=1}^{m}\left(A_{j} d z_{j}+\bar{A}_{j} d \bar{z}_{j}\right), \\
\nabla e_{L} & =\varphi \otimes e_{L}, \quad \text { hence } \nabla e_{L}^{\otimes N}=N \varphi \otimes e_{L}^{\otimes N} .
\end{aligned}
$$

We let $\frac{\partial^{h}}{\partial z_{j}} \in H^{1,0} X$ denote the horizontal lift of $\frac{\partial}{\partial z_{j}}$. The condition $\left(\frac{\partial^{h}}{\partial z_{j}}, \alpha\right)=0$ yields

$$
\frac{\partial^{h}}{\partial z_{j}}=\frac{\partial}{\partial z_{j}}-A_{j} \frac{\partial}{\partial \theta}, \quad \frac{\partial^{h}}{\partial \bar{z}_{j}}=\frac{\partial}{\partial \bar{z}_{j}}-\bar{A}_{j} \frac{\partial}{\partial \theta} .
$$

Suppose $s_{N}=f e_{L}^{\otimes N}$ is a local section of $L^{N}$. Then by (2) and (5),

$$
\hat{s}_{N}(z, \theta)=f(z) a(z)^{-\frac{1}{2}} e^{i N \theta} .
$$

Differentiating (7) and using (3), we conclude that

$$
\begin{aligned}
\varphi & =-\frac{1}{2} d \log a-i \beta \\
& =-\sum_{j=1}^{m}\left(\frac{1}{2} \frac{\partial \log a}{\partial z_{j}}+i A_{j}\right) d z_{j}-\sum_{j=1}^{m}\left(\frac{1}{2} \frac{\partial \log a}{\partial \bar{z}_{j}}+i \bar{A}_{j}\right) d \bar{z}_{j} .
\end{aligned}
$$

Now suppose that $\left(z_{1}, \ldots, z_{m}, \theta\right)$ are Heisenberg coordinates at $P_{0}$; i.e., $e_{L}$ is a preferred frame at $P_{0}$ and $\left(z_{1}, \ldots, z_{m}\right)$ are preferred coordinates centered at $P_{0}$ (with $\left.P_{0}=0\right)$. By property (ii) of preferred frames, we have $\varphi(0)=0$, and hence by (8)

$$
\begin{gathered}
\left.d a\right|_{0}=\left.d \log a\right|_{0}=0, \\
A_{j}(0)=0, \quad(1 \leq j \leq m) .
\end{gathered}
$$


By differentiating (8) and applying the properties of preferred coordinates and frames, we further obtain

$$
\sum_{j=1}^{m} d \bar{z}_{j} \otimes d z_{j}=-\nabla \varphi=\sum_{j=1}^{m} d\left(\frac{1}{2} \frac{\partial \log a}{\partial z_{j}}+i A_{j}\right) \otimes d z_{j}+\sum_{j=1}^{m} d\left(\frac{1}{2} \frac{\partial \log a}{\partial \bar{z}_{j}}+i \bar{A}_{j}\right) \otimes d \bar{z}_{j} \text { at } 0 .
$$

Thus the following four equations are satisfied at $P_{0}=0$ :

$$
\begin{array}{ll}
\frac{1}{2} \frac{\partial^{2} \log a}{\partial z_{j} \partial z_{k}}+i \frac{\partial A_{j}}{\partial z_{k}}=0, & \frac{1}{2} \frac{\partial^{2} \log a}{\partial z_{j} \partial \bar{z}_{k}}+i \frac{\partial A_{j}}{\partial \bar{z}_{k}}=\delta_{k}^{j}, \\
\frac{1}{2} \frac{\partial^{2} \log a}{\partial \bar{z}_{j} \partial z_{k}}+i \frac{\partial \bar{A}_{j}}{\partial z_{k}}=0, & \frac{1}{2} \frac{\partial^{2} \log a}{\partial \bar{z}_{j} \partial \bar{z}_{k}}+i \frac{\partial \bar{A}_{j}}{\partial \bar{z}_{k}}=0
\end{array}
$$

at $P_{0}$. Solving (11) and recalling that $a(0)=1,\left.d a\right|_{0}=0$, we obtain

$$
\begin{aligned}
\frac{\partial^{2} a}{\partial z_{j} \partial z_{k}}(0) & =0, & & \frac{\partial^{2} a}{\partial z_{j} \partial \bar{z}_{k}}(0)=\delta_{k}^{j} \\
\frac{\partial A_{j}}{\partial z_{k}}(0) & =0, & & \frac{\partial A_{j}}{\partial \bar{z}_{k}}=-\frac{i}{2} \delta_{k}^{j} .
\end{aligned}
$$

Hence $A_{j}=-\frac{i}{2} \bar{z}_{j}+O\left(|z|^{2}\right)$ and

$$
\frac{\partial^{h}}{\partial z_{j}}=\frac{\partial}{\partial z_{j}}+\left[\frac{i}{2} \bar{z}_{j}+O\left(|z|^{2}\right)\right] \frac{\partial}{\partial \theta}, \quad \frac{\partial^{h}}{\partial \bar{z}_{j}}=\frac{\partial}{\partial \bar{z}_{j}}-\left[\frac{i}{2} z_{j}+O\left(|z|^{2}\right)\right] \frac{\partial}{\partial \theta} .
$$

\section{Almost holomorphic Sections And Szegö Kernels}

In this section, we define the space $H_{J}^{0}\left(M, L^{N}\right)$ of almost holomorphic sections of $L^{N}$, or equivalently the space $\mathcal{H}_{N}^{2}(X)$ of almost $\mathrm{CR}$ functions in $\mathcal{H}^{2}(X)$ which transform by $e^{i N \theta}$ under the $S^{1}$ action. The Szegö kernels $\Pi_{N}$ are the orthogonal projections onto the spaces $\mathcal{H}_{N}^{2}(X)$.

The order of events in the definition of $H_{J}^{0}\left(M, L^{N}\right)$ is almost opposite to that in the holomorphic case. There, one defines $\mathcal{H}^{2}(X)$ as the kernel of the $\bar{\partial}_{b}$ operator. As mentioned above, the kernel is generically empty in the non-integrable almost complex case. It is correct but somewhat misleading to say that one defines almost holomorphic by deforming $\bar{\partial}_{b}$ in the non-integrable case to a pseudodifferential operator $\bar{D}_{0}$ (which is the zeroth step of a $\bar{D}$ complex), setting $\mathcal{H}^{2}(X)$ equal to its kernel, and defining $\Pi$ as the associated orthogonal projection with respect to a metric on $L$. The misleading aspect is that actually one first defines $\Pi$ as a complex Fourier integral projection operator associated to a certain canonical relation $C$ which naturally generalizes the one in the integrable case. Having defined $\Pi$, one then defines $\bar{D}$ as a deformation of $\bar{\partial}_{b}$ that annihilates $\Pi$.

Existence of a projection $\Pi$ with a prescribed principal symbol in the algebra $I^{*}(X \times X, C)$ of Fourier integral kernels with wave front along $C$ follows from symbolic and functional analytic arguments, but uniqueness does not. Indeed, $\Pi$ is clearly not unique, since it could be replaced by $e^{-i A} \Pi e^{i A}$ for any pseudodifferential operator $A$ of order -1 on $X$. We use the term 'Szegö kernels' rather than 'the Szegö kernel' to remind the reader that $\Pi$ is by no means uniquely defined as in the holomorphic case. The space $H_{J}^{0}\left(M, L^{N}\right)$ therefore also involves non-unique choices. 
The non-geometric construction of the spaces of almost holomorphic sections may seem less odd if one compares it to the construction of asymptotically holomorphic sections in Don1. There, only $\bar{\partial}_{b}$ is defined and asymptotically holomorphic sections are sequences of sections $\left\{s_{N}\right\}$ which are annihilated by $\bar{\partial}_{b}$ modulo small errors. For a fixed power $N$, no space of sections is thereby singled out. On the other hand, our construction gives linear spaces $H_{J}^{0}\left(M, L^{N}\right)$ of almost holomorphic sections, but these spaces depend on the choice of the projection operator $\Pi$.

Let us briefly review the holomorphic case from our perspective. It was proved in [BoSj] that the Szegö kernel of a strictly pseudoconvex domain (including disk bundles of positively curved line bundles) is a complex Fourier integral operator of the form

$$
\Pi(x, y) \sim \int_{\mathbb{R}^{+}} e^{i t \psi(x, y)} s(x, y, t) d t,
$$

with $s \sim \sum_{n=0}^{\infty} t^{m-n} s_{n}(x, y)$ and with $t \psi(x, y)$ a phase of positive type. (Complex Fourier integral operators are discussed below.) Note that the phase is linear in the one-dimensional phase variable $t$. This is a 'hard' result because it shows that an object of complex geometry, the Szegö kernel, has a special singularity structure. It requires estimates of J. J. Kohn-in particular, the closed range property of $\square_{b}$ (cf. [BoSj]).

In BSZ1 , we related $\psi$ to the hermitian metric on $L$, which defines the circle bundle $X$. Let us recall the result. Fix a local holomorphic section $e_{L}$ of $L$ over $U \subset M$ and define $a \in \mathcal{C}^{\infty}(U)$ by $a=\left|e_{L}\right|_{h}^{-2}$. Since $\left.L^{*}\right|_{U} \approx U \times \mathbb{C}$ we can define local coordinates on $L^{*}$ by $(z, \lambda) \approx \lambda e_{L}(z)$. Then a defining function of $X \subset L^{*}$ is given by $\rho(z, \lambda)=1-|\lambda|^{2} a(z)$. Define the function $a(z, w)$ as the almost analytic extension of $a(z)$, i.e. the solution of $\bar{\partial}_{z} a=0=\partial_{w} a, a(z, z)=a(z)$ and put $\psi(x, y)=i(1-\lambda \bar{\mu} a(z, w))$. Then $t \psi$ is a phase for $\Pi$. Because $\Pi$ is a projection, the complex canonical relation $C \subset T^{*}(\tilde{X} \times \tilde{X})$ parametrized by $t \psi$ must be idempotent (i.e., $C^{2}=C=C^{*}$ ). As will be explained below, it is the flow-out of the diagonal in $X \times X$ under the joint Hamiltonian flow of the components of $\bar{\partial}_{b}$, which commute by integrability of the complex structure. Such a flow-out is an equivalence relation ( $x \sim y$ if they belong to the same orbit of the commuting flows), and in this way one also sees that $C$ is idempotent.

Our purpose now is to construct a complex Fourier integral projection kernel $\Pi$ in the almost complex (non-integrable) case with the same properties as in the complex case. As discussed above, this is not the same kind of 'hard' result as in the complex case because there is no a priori definition of $\Pi$ dictated by almost complex geometry, so we simply construct one with analogous microlocal properties. Consequently, we do not need any difficult results of analysis, and the construction involves only symbol algebra and geometry. Indeed, the main step is to define the canonical relation $C$. Since $\bar{\partial}_{b}$ is no longer integrable, we cannot use its components to define the flow-out. Instead, we begin in $\$ 2.2$ by defining a new positive Lagrangean ideal $\mathcal{I}$ corresponding to the ideal generated by the elements of $H^{0,1} X$ in the complex case. We then show in $\$ 2.3$ that $\mathcal{I}$ defines a unique canonical relation $C$ (which lives in the almost analytic extension of $T^{*} X \times T^{*} X$ ) and we describe how $C$ can be parametrized by a phase defined on $X \times X \times \mathbb{R}^{+}$, as in the holomorphic case. In $\oint 2.4$, we describe the algebra $I^{*}(X \times X, C)$ of complex Fourier integral operators with wave front sets along $C$, and construct a Szegö projector $\Pi \in I^{*}(X \times X, C)$. Finally we introduce in $\S 2.5$ the Boutet de Monvel - Guillemin $\bar{D}$ complex of first order pseudodifferential operators with 
ker $\bar{D}_{0}=\mathcal{H}^{2}(X)$. the operator $\bar{D}_{0}$ is the replacement for $\bar{\partial}_{b}$ in the symplectic case, and the $N^{\text {th }}$ Fourier component of $\mathcal{H}^{2}(X)$ is the space $H_{J}^{0}\left(M, L^{N}\right)$ of almost holomorphic sections.

2.1. Description of the algebra $I^{*}(X \times X, C)$. In order to construct an almost holomorphic Szegö kernel, we must first construct the algebra $I^{*}(X \times X, C)$ of complex Fourier integral operators in which it lies. The definition of the algebra $I^{*}(X \times X, C)$ of complex Fourier integral operators is essentially given in Hör (see Definition 25.5.1), although there it is denoted $I^{*}(X \times X, \mathcal{I})$ where $\mathcal{I}$ is a positive conic Lagrangean ideal. (We use the older notation of [MeISj] and the language of almost analytic extensions for ease of comparison with our basic references [MelSj, BoSj], MenSj].) It would take us too far afield to review the definitions and properties of complex Fourier integral operators, so we only briefly recall some basic ideas and refer the reader to [Hö] or [MelSj] for background.

The most intuitive definition of a Fourier integral kernel $A(x, y) \in I^{n}(X \times X, \Lambda)$ is that it is a distribution (generalized function) defined by a complex oscillatory integral

$$
A(x, y)=\int_{\mathbb{R}^{N}} e^{i \varphi(x, y, \theta)} s(x, y, \theta) d \theta
$$

where $\varphi$ is a phase function of positive type parametrizing $\Lambda$, and where $s(x, y, \theta)$ is a symbol of order $n+(\operatorname{dim} X-N) / 2$ (cf., [Hör], Proposition 25.1.5). More precisely, we assume $\varphi(x, y, \theta)$ is a regular phase function in the sense of ([MelSj], Def. 3.5]), i.e. that it has no critical points, is homogeneous of degree one in $\theta$, that the differentials $d \frac{\partial \varphi}{\theta_{j}}$ are linearly independent over $\mathbb{C}$ on the set

$$
C_{\varphi \mathbb{R}}=\left\{(x, y, \theta): d_{\theta} \varphi=0\right\}
$$

and such that $\Im \varphi \geq 0$. We then let $\tilde{\varphi}(\tilde{x}, \tilde{y}, \tilde{\theta})$ be an almost analytic extension of $\varphi$ to $\tilde{X} \times \tilde{X} \times \mathbb{C}^{N}$. Here, $\tilde{Y}$ denotes the almost analytic extension of a $\mathcal{C}^{\infty}$ manifold $Y$. When $Y$ is real analytic, $\tilde{Y}$ is the usual complexification of $Y$, i.e. a complex manifold in which $Y$ sits as a totally real submanifold. The reader may find it simpler to make the extra assumption that $X$ is real analytic. For background on almost analytic extensions, we refer to [MelSj, MenSj].

We put

$$
C_{\tilde{\varphi}}=\left\{(\tilde{x}, \tilde{\theta}): d_{\tilde{\theta}} \tilde{\varphi}=0\right\}
$$

and define the Lagrange immersion

$$
\iota_{\tilde{\varphi}}:(\tilde{x}, \tilde{\theta}) \in C_{\tilde{\varphi}} \rightarrow\left(\tilde{x}, d_{\tilde{x}} \tilde{\varphi}(\tilde{x}, \tilde{\theta})\right) .
$$

We say that the phase $\varphi$ parametrizes $\Lambda$ if $\tilde{\Lambda}$ is the image of this map.

By symbols, we mean the following: the space of classical symbols of order $k$, denoted $S^{k}\left(X \times X \times \mathbb{R}^{N}\right)$, consists of elements of the form $s \sim \sum_{j=0}^{\infty} s_{j}(x, y, \theta)$, where $s_{j}$ is a smooth function (defined near $x=y$ ) of $(x, y)$ and is homogeneous of order $k-j$ in $\theta \in \mathbb{R}^{N}$.

Finally, we note that if $\Lambda$ is an equivalence relation, then $I^{*}(X \times X, \Lambda)$ is an algebra BoSj, Hör. 
2.2. The positive Lagrangean ideal. We begin with some microlocal analysis of $\bar{\partial}_{b}$ in order to introduce the characteristic variety $\Sigma$ of $\bar{\partial}_{b}$. In general, we denote by $\sigma_{A}$ the principal symbol of a pseudodifferential operator $A$. To describe the principal symbol of $\bar{\partial}_{b}$, we introduce convenient local coordinates and frames. Recalling that $H X=H^{1,0} X \oplus H^{0,1} X$, we again consider local orthonormal frames $Z_{1}, \ldots, Z_{n}$ of $H^{1,0} X$, resp. $\bar{Z}_{1}, \ldots, \bar{Z}_{m}$ of $H^{0,1} X$, and dual orthonormal coframes $\vartheta_{1}, \ldots, \vartheta_{m}$, resp. $\bar{\vartheta}_{1}, \ldots, \bar{\vartheta}_{m}$. Then we have $\bar{\partial}_{b}=\sum_{j=1}^{m} \bar{\vartheta}_{j} \otimes$ $\bar{Z}_{j}$. Let us define complex-valued functions on $T^{*} X$ by:

$$
p_{j}(x, \xi)=\left\langle Z_{j}(x), \xi\right), \quad \bar{p}_{j}(x, \xi)=\left\langle\bar{Z}_{j}(x), \xi\right\rangle .
$$

Then

$$
\sigma_{\bar{\partial}_{b}}(x, \xi)=\sum_{j=1}^{m} p_{j}(x, \xi) \epsilon\left(\bar{\vartheta}_{j}\right)
$$

where $\epsilon$ denotes exterior multiplication. We note that $\left\{\bar{p}_{j}, \bar{p}_{k}\right\}=\left\langle\left[\bar{Z}_{j}, \bar{Z}_{k}\right], \xi\right\rangle$.

To state results, it is convenient to introduce the operator $\square_{b}:=\bar{\partial}_{b}^{*} \bar{\partial}_{b}=\sum_{j=1}^{m} \bar{Z}_{j}^{*} \bar{Z}_{j}$ where $\bar{Z}_{j}^{*}$ is the adjoint of the vector field regarded as a linear differential operator. To conform to the notation of [BoGu] we also put $q=\sigma\left(\square_{b}\right)=\sum_{j=1}^{m}\left|\bar{p}_{j}\right|^{2}$. The characteristic variety $\Sigma=\{q=0\}$ of $\bar{\partial}_{b}$ is the same as that of $\square_{b}$, namely the vertical sub-bundle of $T^{*} X \rightarrow M$. It is the conic submanifold of $T^{*} X$ parametrized by the graph of the contact form, $\Sigma=\left\{\left(x, r \alpha_{x}\right): r>0\right\} \sim X \times \mathbb{R}^{+}$. It follows that $\Sigma$ is a symplectic submanifold. It is the dual (real) line bundle to the vertical subbundle $V \subset T X$, since $\alpha(X)=G\left(X, \frac{\partial}{\partial \theta}\right)$.

We are now ready to introduce the positive Lagrangean ideal $\mathcal{I}$ whose generators will define a canonical relation $C$ underlying the Szegö kernels and the principal symbol of the operator $\bar{D}_{0}$, which will replace $\bar{\partial}_{b}$. For background on positive Lagrangean ideals, see [Hör.

Proposition 2.1. There exists a unique positive Lagrangean ideal $\mathcal{I}$ with respect to $\Sigma$ containing $q$. That is, there exists a unique ideal $\mathcal{I} \subset I_{\Sigma}$ (where $I_{\Sigma}$ is the ideal of functions vanishing on $\Sigma$ ) satisfying:

- I is closed under Poisson bracket;

- $\Sigma$ is the set of common zeros of $f \in \mathcal{I}$;

- There exist local generators $\zeta_{1}, \ldots, \zeta_{m}$ such that the matrix $\left(\frac{1}{i}\left\{\zeta_{j}, \bar{\zeta}_{k}\right\}\right)$ is positive definite on $\Sigma$ and that $q=\sum_{j, k} \lambda_{j \bar{k}} \zeta_{j} \bar{\zeta}_{k}$, where $\left\{\lambda_{j \bar{k}}\right\}$ is a hermitian positive definite matrix of functions.

Proof. In the holomorphic case, $\mathcal{I}$ is generated by the linear functions $\zeta_{j}(x, \xi)=\left\langle\xi, \bar{Z}_{j}\right\rangle$. In the general almost complex (or rather almost CR) setting, these functions do not Poisson commute and have to be modified. Since the deviation of an almost complex structure from being integrable (i.e. a true complex structure) is measured by the Nijenhuis bracket, it is not surprising that the generators $\zeta_{j}$ can be constructed from the linear functions $\left\langle\xi, \bar{Z}_{j}\right\rangle$ and from the Nijenhuis tensor. We now explain how to do this, basically following the method of [BoGu].

As a first approximation to the $\zeta_{j}$ we begin with the linear functions $\zeta_{j}^{(1)}=\bar{p}_{j}$ on $T^{*} X$. As mentioned above, the $\zeta_{j}^{(1)}$ do not generate a Lagrangean ideal in the non-integrable almost complex case, indeed

$$
\left\{\zeta_{j}^{(1)}, \zeta_{k}^{(1)}\right\}=\left\langle\xi,\left[\bar{Z}_{j}(x), \bar{Z}_{k}(x)\right]\right\rangle
$$


However we do have that

$$
\left\{\zeta_{j}^{(1)}, \zeta_{k}^{(1)}\right\}=\left\{\left\langle\xi, \bar{Z}_{j}(x)\right\rangle,\left\langle\xi, \bar{Z}_{k}(x)\right\rangle\right\}=0 \text { on } \Sigma .
$$

Indeed, for $(x, \xi) \in \Sigma$, we have $\xi=r \alpha_{x}$ for some $r>0$ so that

$$
\begin{aligned}
& \left\{\left\langle\xi, \bar{Z}_{j}(x)\right\rangle,\left\langle\xi, \bar{Z}_{k}(x)\right\rangle\right\}=r \alpha_{x}\left(\left[\bar{Z}_{j}(x), \bar{Z}_{k}(x)\right]\right) \\
& \quad=r d \alpha_{x}\left(\bar{Z}_{j}(x), \bar{Z}_{k}(x)\right)=r \pi^{*} \omega\left(\bar{Z}_{j}(x), \bar{Z}_{k}(x)\right)=0
\end{aligned}
$$

since $\left\{\bar{Z}_{j}\right\}$ forms a Lagrangean subspace for the horizontal symplectic form $\pi^{*} \omega$. Here, $\pi: X \rightarrow M$ is the natural projection. Moreover if we choose the local horizontal vector fields $Z_{j}$ to be orthonormal relative to $\pi^{*} \omega$, then we also have:

$$
\begin{aligned}
& \left\{\zeta_{j}^{(1)}, \bar{\zeta}_{k}^{(1)}\right\}(x, \xi)=\left\langle\xi,\left[\bar{Z}_{j}(x), Z_{k}(x)\right]\right\rangle=r \pi^{*} \omega\left(\bar{Z}_{j}(x), Z_{k}(x)\right) \\
& \quad=i r \delta_{j}^{k}=i \delta_{j}^{k} p_{\theta}(x, \xi), \quad(x, \xi) \in \Sigma .
\end{aligned}
$$

Here, $p_{\theta}(x, \xi)=\left\langle\xi, \frac{\partial}{\partial \theta}\right\rangle$.

Finally, we have

$$
q=\sum_{j=1}^{m}\left|\left\langle\xi, Z_{j}\right\rangle\right|^{2}=\sum_{j=1}^{m}\left|\zeta_{j}^{(1)}\right|^{2}
$$

Hence the second and third conditions on the $\zeta_{j}$ are satisfied by the functions $\zeta_{j}^{(1)}$. Furthermore, equation (15) tells us that the first condition is satisfied to zero-th order for the ideal $\mathcal{I}_{1}=\left(\zeta_{1}^{(1)}, \ldots, \zeta_{m}^{(1)}\right)$. In fact, let us precisely describe the error. We consider the orthonormal (relative to $\omega$ ) vector fields $Z_{j}^{M}=\pi_{*} Z_{j}$ of type $(1,0)$ on $M$. Recall that the Nijenhuis tensor is given by

Hence,

$$
N(V, W)=\frac{1}{2}([J V, J W]-[V, W]-J[V, J W]-J[J V, W]) .
$$

$$
N\left(Z_{j}^{M}, Z_{k}^{M}\right)=(-1-i J)\left[Z_{j}^{M}, Z_{k}^{M}\right]=-2\left[Z_{j}^{M}, Z_{k}^{M}\right]_{(0,1)} \stackrel{\text { def }}{=} \sum_{p=1}^{m} N_{j k}^{p} \bar{Z}_{p}^{M} .
$$

We note that by definition,

$$
N_{j k}^{p}=N_{k j}^{p}
$$

Furthermore, by the Jacobi identity

$$
\left\{\left\{\zeta_{j}, \zeta_{k}\right\}, \zeta_{p}\right\}+\left\{\left\{\zeta_{p}, \zeta_{j}\right\}, \zeta_{k}\right\}+\left\{\left\{\zeta_{k}, \zeta_{p}\right\}, \zeta_{j}\right\}=0
$$

applied to $\left(x, \alpha_{x}\right) \in \Sigma$, we have

$$
N_{j k}^{p}+N_{p j}^{k}+N_{k p}^{j}=0
$$

By (16) and (18), we have

$$
\left\{\zeta_{j}^{(1)}, \zeta_{k}^{(1)}\right\}=\sum_{p=1}^{m} f_{p}^{1} \zeta_{p}^{(1)}+\sum_{p=1}^{m} \bar{N}_{j k}^{p} \bar{\zeta}_{p}^{(1)}
$$

We now argue, following [BoGu], that these functions can be successively modified to satisfy the same conditions to infinite order on $\Sigma$. The next step is to modify the functions 
$\zeta_{j}^{(1)}$ by quadratic terms so that they satisfy the conditions $\left\{\zeta_{j}, \zeta_{k}\right\} \in \mathcal{I}$ to first order and the condition $q=\sum_{j}\left|\zeta_{j}\right|^{2}$ to order 3 on $\Sigma$. So we try to construct new functions

$$
\zeta_{p}^{(2)}=\zeta_{p}^{(1)}+R_{p}, \quad R_{p}=\sum_{j, k} \nu_{p}^{j k} \zeta^{\overline{(1)}}{ }_{j} \zeta^{(1)}{ }_{k}
$$

so that

$$
\begin{aligned}
& \left\{\zeta_{j}^{(2)}, \zeta_{k}^{(2)}\right\}=\sum_{p} f_{p}^{2} \zeta_{p}^{(2)}+\sum_{\alpha_{1}, \alpha_{2}} \mu_{j k}^{\alpha_{1} \alpha_{2}} \bar{\zeta}^{(2)}{ }_{\alpha_{1}} \zeta^{\overline{(2)}}{ }_{\alpha_{2}} \\
& q=\sum_{p} v_{p}^{2} \zeta_{p}^{(2)}+\sum_{\alpha} \varphi_{p}^{\alpha} \bar{\zeta}^{\overline{(2)}}{ }_{\alpha_{1}} \zeta^{\overline{(2)}}{ }_{\alpha_{2}} \bar{\zeta}^{\overline{(2)}}{ }_{\alpha_{3}} \zeta^{\overline{(2)}}{ }_{\alpha_{4}}, \quad\left(\alpha=\left(\alpha_{1}, \ldots, \alpha_{4}\right)\right) .
\end{aligned}
$$

Let us now solve (22)-(23) for the $\nu_{p}^{j k}$. First of all, we choose $\nu_{p}^{j k}=\nu_{p}^{k j}$. We have

$$
\left\{\zeta_{j}^{(2)}, \zeta_{k}^{(2)}\right\}=\sum_{p=1}^{m} f_{p}^{1} \zeta_{p}^{(1)}+\sum_{p=1}^{m} \bar{N}_{j k}^{p} \zeta^{\overline{(1)}}+\left\{\zeta_{j}^{(1)}, R_{k}\right\}-\left\{\zeta_{k}^{(1)}, R_{j}\right\} \quad \bmod I_{\Sigma}^{2}
$$

By (17), we have

$$
\left\{\zeta_{j}^{(1)}, \bar{\zeta}^{(1)}{ }_{k}\right\}=i \delta_{j}^{k} p_{\theta} \bmod I_{\Sigma}
$$

and thus

$$
\left\{\zeta_{j}^{(1)}, R_{k}\right\}=\sum_{p=1}^{m} 2 i \nu_{k}^{p j} p_{\theta} \zeta^{\overline{(1)}} \quad \bmod I_{\Sigma}^{2}
$$

Therefore,

$$
\left\{\zeta_{j}^{(2)}, \zeta_{k}^{(2)}\right\}=\sum_{p=1}^{m} f_{p}^{1} \zeta_{p}^{(2)}+\sum_{p=1}^{m}\left(\bar{N}_{j k}^{p}+2 i\left(\nu_{k}^{p j}-\nu_{j}^{p k}\right) p_{\theta}\right) \zeta_{p}^{(1)} \quad \bmod I_{\Sigma}^{2}
$$

Hence

$$
\bar{N}_{j k}^{p}=2 i\left(\nu_{j}^{p k}-\nu_{k}^{p j}\right) p_{\theta} \quad \text { on } \Sigma
$$

or equivalently,

$$
\nu_{j}^{p k}-\nu_{k}^{p j}=\frac{i}{2 p_{\theta}} \bar{N}_{j k}^{p} \quad \bmod I_{\Sigma}
$$

On the other hand,

$$
\begin{aligned}
q & =\sum_{p}\left|\zeta_{p}^{(2)}-R_{p}\right|^{2}=\sum_{p} v_{p}^{2} \zeta_{p}^{(2)}-R_{p} \zeta^{\overline{(2)}}{ }_{p} \\
& =\sum_{p} v_{p}^{2} \zeta_{p}^{(2)}-\sum_{j, k, p} \nu_{p}^{j k} \zeta^{(2)}{ }_{j} \zeta^{(2)}{ }_{k} \zeta^{(2)}{ }_{p}+\sum_{\alpha} \varphi_{p}^{\alpha} \bar{\zeta}^{(2)}{ }_{\alpha_{1}} \zeta^{(2)}{ }_{\alpha_{2}} \bar{\zeta}^{(2)}{ }_{\alpha_{3}} \zeta^{\overline{(2)}}{ }_{\alpha_{4}}
\end{aligned}
$$

Hence (23) is equivalent to

$$
\nu_{p}^{j k}+\nu_{k}^{p j}+\nu_{j}^{k p}=0 .
$$

Using (19)-(20), we can solve the equations (24) and (26) to obtain

$$
\nu_{p}^{j k}=\frac{i}{6 p_{\theta}}\left(\bar{N}_{p j}^{k}+\bar{N}_{p k}^{j}\right) .
$$


Indeed, the solution (28) is unique (modulo $I_{\Sigma}$ ) and hence the $R_{p}$ are unique modulo $I_{\Sigma}^{3}$. In summary,

$$
\zeta_{p}^{(2)}=\zeta_{p}^{(1)}+\frac{i}{3 p_{\theta}} \sum_{j, k} \bar{N}_{p j}^{k} \zeta^{\overline{(1)}{ }_{j}} \zeta_{k}^{(1)}{ }_{k}
$$

The passage from the $n^{\text {th }}$ to the $(n+1)^{\text {st }}$ step is similar, and we refer to BoGu, pp. 147$149]$.

Remark: Define $p_{\theta}(x, \xi)=\left\langle\xi, \frac{\partial}{\partial \theta}\right\rangle$. Since the joint zero set of $\left\{\zeta_{1}, \ldots, \zeta_{m}\right\}$ equals $\Sigma$ and since $p_{\theta} \neq 0$ on $\Sigma-0$ it follows that $\left\{\zeta_{1}, \ldots, \zeta_{m}, p_{\theta}\right\}$ is an elliptic system of symbols.

2.3. The complex canonical relation. We now construct the underlying complex canonical relation $C$ and we show that $C$ can be parametrized by a phase of the form $t \psi(x, y)$ as in the complex case (Theorem 2.3).

As mentioned above, $C$ does not live in $T^{*} X \times T^{*} X$ but rather in its almost analytic extension $T^{*} \tilde{X} \times T^{*} \tilde{X}$. Since $\pi: X \rightarrow M$ is an $S^{1}$ bundle over $M$, its complexification $\tilde{\pi}: \tilde{X} \rightarrow \tilde{M}$ defines a $\mathbb{C}^{*}$ bundle over $\tilde{M}$. The connection form $\alpha$ has an (almost) analytic continuation to a connection $\tilde{\alpha}$ to this bundle and we may split $T \tilde{X}=\tilde{H} \oplus \tilde{V}$, where $\tilde{V} \rightarrow T \tilde{M}$ is the vertical subbundle of the fibration $\tilde{X} \rightarrow \tilde{M}$ and where $\tilde{H} \rightarrow T \tilde{M}$ is the kernel of $\tilde{\alpha}$.

The (almost) complexification of $T^{*} X$ is of course $T^{*}(\tilde{X})$. We denote the canonical symplectic form on $T^{*} X$ by $\sigma$ and that on $T^{*}(\tilde{X}$ by $\tilde{\sigma}$; the notation is consistent because it is the complexification of $\sigma$. The symplectic cone $\Sigma$ complexifies to $\tilde{\Sigma}$ and it remains symplectic with respect to $\tilde{\sigma}$. It is given by $\left\{\left(\tilde{x}, \tilde{\lambda} \tilde{\alpha}_{\tilde{x}}\right): \tilde{\lambda} \in \mathbb{C}^{*}\right\}$. We have a natural identification $L^{*} \Longleftrightarrow \Sigma$ given by $r x \rightarrow\left(x, r \alpha_{x}\right)$. We further note that the $\mathbb{C}^{*}$ bundle $L^{*} \rightarrow M$ is the fiberwise complexification of the $S^{1}$ bundle $X \rightarrow M$, hence $L^{*} \rightarrow M$ is the restriction of $\tilde{\pi}$ to $\tilde{\pi}^{-1}(M)$. We will therefore view $L^{*}$ as a submanifold of $\tilde{X}$.

We let $\tilde{\zeta}_{j}$ be almost analytic extensions to $\tilde{X}$ of the functions $\zeta_{j}$. We then define

$$
\mathcal{J}_{+}=\left\{(\tilde{x}, \tilde{\xi}) \in T^{*} \tilde{X}: \tilde{\zeta}_{j}=0 \forall j\right\},
$$

which is an involutive manifold of $T^{*} \tilde{X}$ with the properties:

$$
\begin{aligned}
& \text { (i) }\left(\mathcal{J}_{+}\right)_{\mathbb{R}}=\Sigma \\
& \text { (ii) }\left.q\right|_{\mathcal{J}_{+}} \sim 0 \\
& \text { (iii) } \frac{1}{i} \sigma(u, \bar{u})>0, \forall u \in T\left(\mathcal{J}_{+}\right)^{\perp} \\
& \text { (iv) } T_{\rho}\left(\mathcal{J}_{+}\right)=T_{\rho} \tilde{\Sigma} \oplus W_{\rho}^{+} .
\end{aligned}
$$

Here, $W_{\rho}^{+}$is the sum of the eigenspaces of $F_{\rho}$, the normal Hessian of $q$, corresponding to the eigenvalues $\left\{i \lambda_{j}\right\}$. The null foliation of $\mathcal{J}_{+}$is given by the joint Hamilton flow of the $\tilde{\zeta}_{j}$ 's.

The following proposition, proved in [MenSj] and in (BoGu]), Appendix, Lemma 4.5), adapts to our context and defines the complex canonical relation $C$ :

Proposition 2.2. There exists a unique strictly positive almost analytic canonical relation $C$ satisfying

$$
\operatorname{diag}(\Sigma) \subset C \subset \mathcal{J}_{+} \times \overline{\mathcal{J}_{+}}
$$


Indeed,

$$
C=\left\{(\tilde{x}, \tilde{\xi}, \tilde{y}, \tilde{\eta}) \in \mathcal{J}_{+} \times \overline{\mathcal{J}_{+}}:(\tilde{x}, \tilde{\xi}) \sim(\tilde{y}, \tilde{\eta})\right\},
$$

where $\sim$ is the equivalence relation of 'belonging to the same leaf of the null foliation of $\mathcal{J}_{+}$. Thus, $C$ is the flow-out of its real points, $\operatorname{diag}(\Sigma)$, under the joint Hamilton flow of the $\tilde{\zeta}_{j}$ 's. It is clear from the description that $C \circ C=C^{*}=C$, i.e. that $C$ is an idempotent canonical relation.

We now prove that $C$ can be parametrized by a phase $t \psi(x, y)$ defined on $X \times X \times \mathbb{R}^{+}$. This is helpful in analyzing the scaling limit of $\Pi_{N}(x, y)$. In the following we use local coordinates $(z, \lambda)$ on $L^{*}$ coming from a choice of local coordinates $z$ on $M$ and a local frame $e_{L}(z)$ of $L$, and a corresponding local trivialization $(\tilde{z}, \lambda)$ of $\tilde{X} \rightarrow \tilde{M}$. As before, we let $a=\left\|e_{L}^{*}\right\|^{2}$.

THEOREM 2.3. There exists a unique regular phase function $t \psi(x, y) \in \mathcal{C}^{\infty}\left(X \times X \times \mathbb{R}^{+}\right)$of positive type such that:

- $\left.i d_{x} \psi\right|_{x=y}=-\left.i d_{y} \psi\right|_{x=y}=\alpha$;

- The almost analytic extension $\tilde{\psi} \in \mathcal{C}^{\infty}(\tilde{X} \times \tilde{X})$ of $\psi$ has the form $\tilde{\psi}(\tilde{x}, \tilde{y})=i(1-$ $\lambda \bar{\mu} \tilde{a}(\tilde{z}, \tilde{w}))$ with $\tilde{a}(z, z)=a(z)$ and $\tilde{a}(\tilde{z}, \tilde{w})=\overline{\tilde{a}(\tilde{w}, \tilde{z})}$;

- $t \tilde{\psi}$ parametrizes $C$.

Proof. We need to construct a function $a(z, w)$ so that $t \tilde{\psi}$ as above parametrizes the canonical relation $C$, i.e. that $C$ is the image of the Lagrange immersion

$$
\begin{aligned}
& \iota_{\tilde{\psi}}: C_{t \tilde{\psi}}=\mathbb{R}^{+} \times\{\tilde{\psi}=0\} \rightarrow T^{*}(\tilde{X} \times \tilde{X}) \\
& (t, \tilde{x}, \tilde{y}) \mapsto\left(\tilde{x}, t d_{\tilde{x}} \tilde{\psi} ; \tilde{y},-t d_{\tilde{y}} \tilde{\psi}\right)
\end{aligned}
$$

Since $C$ is the unique canonical relation satisfying $\operatorname{diag}(\Sigma) \subset C \subset \mathcal{J}_{+} \times \overline{\mathcal{J}_{+}}$, the conditions that $t \tilde{\psi}$ parametrize $C$ are the following:

i) $\{(x, y) \in X \times X: \psi(x, y)=0\}=\operatorname{diag}(X)$;

ii) $\left.d_{x} \psi\right|_{x=y}=-\left.d_{y} \psi\right|_{x=y}=r \alpha$ for $x, y \in X$ and for some function $r(x)>0$;

iii) $\tilde{\zeta}_{j}\left(\tilde{x}, d_{\tilde{x}} \tilde{\psi}\right)=0=\tilde{\zeta}_{j}\left(\tilde{y}, d_{\tilde{y}} \tilde{\psi}\right)$ on $\{\tilde{\psi}=0\}$.

Such a $\tilde{\psi}$ is not unique, so we require that $r \equiv 1$ in condition (ii), i.e.,

$$
\left.d_{x} \psi\right|_{x=y}=-\left.d_{y} \psi\right|_{x=y}=\alpha .
$$

Suppose we have $\tilde{\psi}(\tilde{x}, \tilde{y})=i(1-\lambda \bar{\mu} \tilde{a}(\tilde{z}, \tilde{w}))$. We observe that

$$
\tilde{\psi}=0 \Longleftrightarrow \tilde{a}(\tilde{z}, \tilde{w})=(\lambda \bar{\mu})^{-1},
$$

and hence

$$
\begin{aligned}
& i d_{\tilde{x}} \tilde{\psi}=\bar{\mu} \tilde{a}(\tilde{z}, \tilde{w}) d \lambda+\lambda \bar{\mu} d_{\tilde{z}} \pi^{*} \tilde{a}(\tilde{z}, \tilde{w}) \\
& \quad=\lambda^{-1} d \lambda+\tilde{a}^{-1} d_{\tilde{z}} \tilde{a}(\tilde{z}, \tilde{w}) \quad \Longleftrightarrow \tilde{\psi}=0 .
\end{aligned}
$$


The conditions on $a$ are therefore:

$$
\left\{\begin{array}{l}
a(z, w) \lambda \bar{\mu}=1 \Longleftrightarrow(z, \lambda)=(w, \mu) \in X ; \\
\left.\left(a^{-1} d_{z} a+\lambda^{-1} d \lambda\right)\right|_{\operatorname{diag}(X)}=-\left.\left(a^{-1} d_{w} a+\lambda^{-1} d \lambda\right)\right|_{\operatorname{diag}(X)}=\alpha \\
\tilde{\zeta}_{j}\left(\tilde{z}, \lambda, \lambda^{-1} d \lambda+\tilde{a}^{-1} d_{\tilde{z}} \tilde{a}(\tilde{z}, \tilde{w})\right)=0=\tilde{\zeta}_{j}\left(\tilde{w}, \tilde{\mu}, \mu^{-1} d \mu+\tilde{a}^{-1} d_{\tilde{w}} \tilde{a}(\tilde{z}, \tilde{w})\right), \forall(z, w, \lambda, \mu)
\end{array}\right.
$$

A solution $a(z, w)$ satisfying the first condition must satisfy $a(z, z)|\lambda|^{2}=1$ on $X$, so that $a(z, z)|\lambda|^{2}$ is the local hermitian metric on $L^{*}$ with unit bundle $X$, i.e. $a(z, z)=a(z)$.

We now prove that these conditions have a unique solution near the diagonal. We do this by reducing the canonical relation $C$ by the natural $S^{1}$ symmetry. The reduced relation $C_{r}$ has a unique generating function $\log a$; the three conditions above on $a$ will follow automatically from this fact.

The $S^{1}$ action of $X$ lifts to $T^{*} X$ as the Hamiltonian flow of the function $p_{\theta}(x, \xi):=\left\langle\xi, \frac{\partial}{\partial \theta}\right\rangle$. The $\zeta_{j}$ are invariant under this $S^{1}$ action, hence

$$
\left\{p_{\theta}, \zeta_{j}\right\}=0 \forall j
$$

Now consider the level set $\left\{p_{\theta}=1\right\} \subset T^{*} X$. Dual to the splitting $T X=H \oplus V$ we get a splitting $T^{*} X=H^{*} \oplus V^{*}$, where

$$
V^{*}(X)=\mathbb{R} \alpha=H^{o}, \quad H^{*}(X)=V^{o}
$$

where $E^{o}$ denotes the annihilator of a subspace $E$, i.e. the linear functionals which vanish on $E$. Thus, $p_{\theta}=0$ on the horizontal space $H^{*}(X)$ and $p_{\theta}(\alpha)=1$. Since $p_{\theta}$ is linear on the fibers of $T^{*} X$, the set $\left\{p_{\theta}=1\right\}$ has the form $\left\{\alpha+h: h \in H^{*}(X)\right\}$. We also note that $p_{\theta}(d \theta)=1$ in the local coordinates $(z, \theta)$ on $X$ defined by $\lambda=e^{i \theta}$. Hence $\left\{p_{\theta}=1\right\}$ may also be identified with $\left\{d \theta+h: h \in H^{*}(X)\right\}$.

Since $\left\{p_{\theta}=1\right\}$ is a hypersurface, its null-foliation is given by the orbits of the Hamiltonian flow of $p_{\theta}$, i.e. by the $S^{1}$ action. We use the term 'reducing by the $S^{1}$-action' to mean setting $p_{\theta}=1$ and then dividing by this action. The reduction of $T^{*} X$ is thus defined by $\left(T^{*} X\right)_{r}=p_{\theta}^{-1}(1) / S^{1}$. Since $p_{\theta}^{-1}(1)$ is an affine bundle over $X$ with fiber isomorphic to $H^{*}(X) \approx T^{*} M$, it is clear that $\left(T^{*} X\right)_{r} \approx T^{*} M$ as vector bundles over $M$. We can obtain a symplectic equivalence using the local coordinates $(z, \theta)$ on $X$. Let $\left(p_{z}, p_{\theta}\right)$ be the corresponding symplectically dual coordinates, so that the natural symplectic form $\sigma_{T^{*} X}$ on $T^{*} X$ is given by $\sigma_{T^{*} X}=d z \wedge d p_{z}+d \theta \wedge d p_{\theta}$. The notation $p_{\theta}$ is consistent with the above. Moreover, the natural symplectic form on $T^{*} M$ is given locally by $\sigma_{T^{*} M}=d z \wedge d p_{z}$. Now define the projection

$$
\chi: p_{\theta}^{-1}(1) \rightarrow T^{*} M, \quad \chi\left(z, p_{z}, 1, p_{\theta}\right)=\left(z, p_{z}\right) .
$$

This map commutes with the $S^{1}$ action and hence descends to the quotient to define a local map over $U$, still denoted $\chi$, from $\left(T^{*} X\right)_{r} \rightarrow T^{*} M$. Clearly $\chi$ is symplectic.

We now reduce the canonical relation $C$. Thus we consider the $\mathbb{C}^{*} \times \mathbb{C}^{*}$ action on $T^{*} \tilde{X} \times$ $T^{*} \tilde{X}-0$ generated by $p_{\theta}(x, \xi), p_{\theta}(y, \eta)$. The reduction of $C$ is given by

$$
C_{r}=C \cap\left(p_{\theta} \times p_{\theta}\right)^{-1}(1,1) / \mathbb{C}^{*} \times \mathbb{C}^{*}
$$


We then use $\chi \times \chi$ to identify $C_{r}$ with a (non-homogeneous) positive canonical relation in $T^{*}(\tilde{M} \times \tilde{M})$. Thus in coordinates,

$$
C_{r}=\left\{\left(\tilde{z}, \tilde{p_{z}}, \tilde{w}, \tilde{p_{w}}\right) \in T^{*}(\tilde{M} \times \tilde{M}): \exists \lambda, \mu,\left(\tilde{z}, \lambda, \tilde{p_{z}}, 1 ; \tilde{w}, \mu, \tilde{p_{w}}, 1\right) \in C\right\} .
$$

Since reduction preserves real points, it is clear that

$$
\begin{aligned}
& \left(C_{r}\right)_{\mathbb{R}}=C_{\mathbb{R}} \cap\left(p_{\theta} \times p_{\theta}\right)^{-1}(1,1) / \mathbb{C}^{*} \times \mathbb{C}^{*} \\
& =\left\{\left(z, p_{z}, z, p_{z}\right) \in \operatorname{diag}\left(T^{*}(M \times M)\right): \exists \theta \text { such that } \alpha_{z, e^{i \theta}}=d \theta+p_{z}\right\} .
\end{aligned}
$$

Let us denote by $\tilde{\zeta}_{j r}$ the reductions of the functions $\tilde{\zeta}_{j}$ by the $S^{1}$ symmetry. Then $\tilde{\zeta}_{j r}=0$ on either pair of cotangent vectors in $C_{r}$. Moreover, by the uniqueness statement on $C$ it follows that $C_{r}$ is the unique canonical relation in $T^{*}(\tilde{M} \times \tilde{M})$ with the given set of real points and in the zero set of the $\tilde{\zeta}_{j r}$ 's.

We now observe that $C_{r}$ has, at least near the diagonal, a unique global generating function. This holds because the natural projection

$$
C_{r} \subset T^{*}(\tilde{M} \times \tilde{M}) \rightarrow \tilde{M} \times \tilde{M}
$$

is a local diffeomorphism near the diagonal. Indeed, its derivative gives a natural isomorphism

$$
T_{\rho, \rho} C_{r} \approx H^{*} \oplus H^{*} \approx T(\tilde{M} \times \tilde{M}) .
$$

Therefore, there exists a global generating function $\log \tilde{a} \in \mathcal{C}^{\infty}(\tilde{M} \times \tilde{M})$ i.e.

$$
C_{r}=\left\{\left(\tilde{z}, d_{\tilde{z}} \log \tilde{a}, \tilde{w}, d_{\tilde{w}} \log \tilde{a}\right), \tilde{z}, \tilde{w} \in \tilde{M}\right\} .
$$

Since $C^{*}=C$ it follows that $C_{r}^{*}=C^{r}$ and hence that $a(w, z)=\overline{a(z, w)}$.

Working backwards, we find that the function $\tilde{\psi}(\tilde{x}, \tilde{y})=i(1-\lambda \bar{\mu} \tilde{a}(\tilde{z}, \tilde{w}))$ satisfies the equations $\tilde{\zeta}_{j}\left(\tilde{x}, d_{\tilde{x}} \tilde{\psi}\right)=\tilde{\zeta}_{j}\left(\tilde{y}, d_{\tilde{y}} \tilde{\psi}\right)=0$ on $\tilde{\psi}=0$. Therefore the Lagrange immersion

$$
\begin{aligned}
& i_{\tilde{\psi}}: C_{t \tilde{\psi}}=\mathbb{R}^{+} \times\{\tilde{\psi}=0\} \rightarrow T^{*}(\tilde{X} \times \tilde{X}) \\
& (t, \tilde{x}, \tilde{y}) \rightarrow\left(\tilde{x}, t d_{\tilde{x}} \tilde{\psi} ; \tilde{y},-t d_{\tilde{y}} \tilde{\psi}\right)
\end{aligned}
$$

takes its image inside $\mathcal{J}_{+} \times \overline{\mathcal{J}_{+}}$and reduces to $C_{r}$ under the $S^{1}$-symmetry. To conclude the proof it is only necessary to show that the real points of the image of $i_{\tilde{\psi}}$ equal $\operatorname{diag}(\Sigma)$. We know however that these real points reduce to $\left(C_{r}\right)_{\mathbb{R}}$ and hence that $z=w$ at real points. But we have

$$
1=\lambda \bar{\mu} a(z, w)=e^{i(\theta-\varphi)} \frac{a(z, w)}{\sqrt{a(z)} \sqrt{a(w)}}, \quad \text { on }\{\tilde{\psi}=0\}
$$

hence when $z=w$ we have $e^{i(\theta-\varphi)}=1$ and hence $x=y$. Since $\left.d_{\tilde{x}} \tilde{\psi}(x, y)\right|_{x=y}=\alpha_{x}$, it follows that the real points indeed equal $\operatorname{diag}(\Sigma)$. Therefore $t \tilde{\psi}$ parametrizes $C$.

To show that the phase is of positive type, we need to describe the asymptotics of $a(z, w)$ near the diagonal. Note that in the almost-complex case, we cannot describe $a(z, w)$ as the almost analytic extension of $a(z, z)$. (Of course, $\tilde{a}(\tilde{z}, \tilde{w})$ is the almost analytic extension of $a(z, w)$, by definition.) For our near-diagonal asymptotics in the nonintegrable case, we instead use the following second order expansion of $a$ at points on the diagonal: 
LEMMA 2.4. Suppose that $\left(z_{1}, \ldots, z_{m}\right)$ are preferred coordinates and $e_{L}$ is a preferred frame at a point $P_{0} \in M$. Then the Taylor expansion of $a(z, w)$ at $z=w=0$ is

$$
a(z, w)=1+z \cdot \bar{w}+\cdots
$$

Proof. To begin, we recall that $a(0,0)=a(0)=\left\|e_{L}^{*}\left(P_{0}\right)\right\|^{2}=1$. To compute the first and second order terms, we return to the equation

$$
\zeta_{j}\left(z, \lambda, \frac{d \lambda}{\lambda}+d_{z} \log a(z, w)\right)=0, \quad \forall(z, \lambda ; w) \in X \times M .
$$

Let us write $\zeta_{j}=\zeta_{j}^{(1)}+R_{j}^{(2)}$, where $R_{j}^{(2)}$ vanishes to second order on $\Sigma$ and we recall that $\zeta_{j}^{(1)}(\xi)=\left(\bar{Z}_{j}, \xi\right)$. Let us also Taylor expand $\log a$ :

$$
\log a=L(z, w)+Q(z, w)+\cdots,
$$

where $L$ is linear and $Q$ is quadratic. Since $e_{L}$ is a preferred frame at $P_{0}$, it follows from (田) that $a(z, z)=1+|z|^{2}+\cdots$ and hence

$$
L(z, z)=0, \quad Q(z, z)=|z|^{2} .
$$

Since $\left.d_{z} \log a\right|_{z=w}+\frac{d \lambda}{\lambda}=\alpha \in \Sigma$, it follows from (41) that

$$
\zeta_{j}^{(1)}\left(z, \lambda, \frac{d \lambda}{\lambda}+d_{z} \log a\right)=-R_{j}^{(2)}\left(z, \lambda, \frac{d \lambda}{\lambda}+d_{z} \log a\right)=O\left(|z-w|^{2}\right) .
$$

Since $a(z, w)=\overline{a(w, z)}$, we can write

$$
L(z, w)=\sum_{j=1}^{m}\left(b_{j} z_{j}+c_{j} \bar{z}_{j}+\bar{c}_{j} w_{j}+\bar{b}_{j} \bar{w}_{j}\right) .
$$

Since the $z_{j}$ are preferred coordinates and $e_{L}$ is a preferred frame at $P_{0}$, we can choose the $\bar{Z}_{j}$ so that $\bar{Z}_{j}(0)=\frac{\partial}{\partial \bar{z}_{j}}$ and hence by 43 ,

$$
0=\left.\zeta_{j}^{(1)}\left(z, \lambda, \frac{d \lambda}{\lambda}+d_{z} \log a\right)\right|_{z=w=0, \lambda=1}=\left.\left(\frac{\partial}{\partial \bar{z}_{j}}, d_{z} \log a\right)\right|_{(0,0)}=c_{j} \forall j .
$$

Since $L(z, z)=0$, we have $b_{j}+\bar{c}_{j}=0$, and hence $L=0$.

To investigate the quadratic term $Q$ in (42), we write

$$
\left.\left(\frac{d \lambda}{\lambda}+d_{z} \log a\right)\right|_{(z, w)}=\alpha_{z}+\sum_{j=1}^{m}\left[z_{j} U_{j}^{\prime}+\bar{z}_{j} U_{j}^{\prime \prime}+w_{j} V_{j}^{\prime}+\bar{w}_{j} V_{j}^{\prime \prime}\right]+O\left(|z|^{2}+|w|^{2}\right),
$$

where

$$
\begin{aligned}
& U_{j}^{\prime}=\sum_{k=1}^{m}\left(\frac{\partial^{2} Q}{\partial z_{j} \partial z_{k}} d z_{k}+\frac{\partial^{2} Q}{\partial z_{j} \partial \bar{z}_{k}} d \bar{z}_{k}\right), \quad U_{j}^{\prime \prime}=\sum_{k=1}^{m}\left(\frac{\partial^{2} Q}{\partial \bar{z}_{j} \partial z_{k}} d z_{k}+\frac{\partial^{2} Q}{\partial \bar{z}_{j} \partial \bar{z}_{k}} d \bar{z}_{k}\right), \\
& V_{j}^{\prime}=\sum_{k=1}^{m}\left(\frac{\partial^{2} Q}{\partial w_{j} \partial z_{k}} d z_{k}+\frac{\partial^{2} Q}{\partial w_{j} \partial \bar{z}_{k}} d \bar{z}_{k}\right), \quad V_{j}^{\prime \prime}=\sum_{k=1}^{m}\left(\frac{\partial^{2} Q}{\partial \bar{w}_{j} \partial z_{k}} d z_{k}+\frac{\partial^{2} Q}{\partial \bar{w}_{j} \partial \bar{z}_{k}} d \bar{z}_{k}\right) .
\end{aligned}
$$

Applying $\zeta_{k}^{(1)}$ to (44) and using (43) and the fact that $\zeta_{k}^{(1)}\left(\alpha_{z}\right)=0$, we have

$$
\sum_{j=1}^{m}\left[z_{j}\left(\left.\bar{Z}_{k}\right|_{z}, U_{j}^{\prime}\right)+\bar{z}_{j}\left(\left.\bar{Z}_{k}\right|_{z}, U_{j}^{\prime \prime}\right)+w_{j}\left(\left.\bar{Z}_{k}\right|_{z}, V_{j}^{\prime}\right)+\bar{w}_{j}\left(\left.\bar{Z}_{k}\right|_{z}, V_{j}^{\prime \prime}\right)\right]=O\left(|z|^{2}+|w|^{2}\right) .
$$


By (11) and (阿),

$$
\left.\bar{Z}_{k}\right|_{z}=\frac{\partial}{\partial \bar{z}_{k}}+\sum_{l=1}^{m} B_{k l}(z) \frac{\partial}{\partial z_{l}}+C_{k}(z) \frac{\partial}{\partial \theta}, \quad B_{k l}(0)=0 .
$$

Hence by $(45)$,

$$
\frac{\partial^{2} Q}{\partial z_{j} \partial \bar{z}_{k}}=\left(\frac{\partial}{\partial \bar{z}_{k}}, U_{j}^{\prime}\right)=\left(\left.\bar{Z}_{k}\right|_{0}, U_{j}^{\prime}\right)=0 .
$$

Similarly, $\frac{\partial^{2} Q}{\partial \bar{z}_{j} \partial \bar{z}_{k}}=\frac{\partial^{2} Q}{\partial w_{j} \partial \bar{z}_{k}}=\frac{\partial^{2} Q}{\partial \bar{w}_{j} \partial \bar{z}_{k}}=0$. Thus $Q(z, w)$ has no terms containing $\bar{z}_{k}$. Since $Q(z, w)=\overline{Q(w, z)}$, the quadratic function $Q$ also has no terms containing $w_{k}$, so we can write

$$
Q(z, w)=B(z, z)+H(z, \bar{w})+\overline{B(w, w)}
$$

where $B$, resp. $H$, is a bilinear, resp. hermitian, form on $\mathbb{C}^{m}$. Since $Q(z, z)=|z|^{2}$ (recall (42)), we conclude that $B(z, z)=0$ and hence $Q(z, w)=H(z, \bar{w})=z \cdot \bar{w}$.

We now complete the proof that the phase is of positive type; i.e., $\Im \psi \geq 0$ on some neighborhood of the diagonal in $X \times X$. Let $x \in X$ be arbitrary and choose Heisenberg coordinates $(z, \theta)$ at $P_{0}=\pi(x)$ (so that $x$ has coordinates $(0,0)$ ). Recalling that $\lambda=$ $a(z)^{-\frac{1}{2}} e^{i \theta}$ on $X$, we have by Lemma 2.4,

$$
\frac{1}{i} \psi(0,0 ; z, \theta)=1-\frac{a(0, z)}{\sqrt{a(z)}} e^{-i \theta}=\left(1-e^{-i \theta}\right)+e^{-i \theta}\left[\frac{1}{2}|z|^{2}+O\left(|z|^{3}\right)\right] \text {. }
$$

Thus,

$$
\Re\left[\frac{1}{i} \psi(0,0 ; z, \theta)\right] \geq 0 \text { for }|\theta|<\frac{\pi}{2},|z|<\varepsilon,
$$

where $\varepsilon$ is independent of the point $P_{0} \in M$.

2.4. The Szegö projector. Having defined $C$, we denote by $I^{*}(X \times X, C)$ the space of complex Fourier integral operators with wave fronts along $C$, as described in $\$ 2.1$. We define a Szegö projector $\Pi$ associated to $\Sigma$ and $C$ to be a self-adjoint projection $\Pi \in I^{0}(X \times X, C)$ with principal symbol $\sigma_{\Pi}$ equal to the canonical 1/2-density of $C$. Our purpose now is to describe the method of [BoSj, §4] [BoGu (Appendix) for producing a canonical Szegö projector modulo finite rank operators. We refer there for further discussion of the method.

Since by Theorem 2.3, $C$ is parametrized by a function of the form $t \psi(x, y)$, the space $I^{n}(X \times X, C)$ consists of Fourier integral operators of the form

$$
\int_{0}^{\infty} e^{i t \psi(x, y)} s(x, y, t) d t, \quad s \sim \sum_{k=0}^{\infty} t^{m+n-k} s_{k}(x, y) \in S^{m+n}\left(X \times X \times \mathbb{R}^{+}\right),
$$

where $s_{k}$ is a smooth function of $(x, y)$ defined near $x=y$. Because $C$ is an equivalence relation, $I^{*}(X \times X, C)$ is a $*$-algebra. Hence there is an induced algebra product (i.e., a *-product) defined on the amplitudes by

$$
I\left(q_{1}, \psi\right) \circ I\left(q_{2}, \psi\right) \sim I\left(q_{1} * q_{2}, \psi\right)
$$

where $\sim$ means roughly that the difference is a smoothing operator. To be more precise, one uses the method of stationary phase as in [BoSj], §4, (4.14)] to determine the symbol expansion of the $*$-product $*: I^{n_{1}} \times I^{n_{2}} \rightarrow I^{n_{1}+n_{2}}$ as a formal infinite series. We do not wish 
to consider its convergence, hence we will use $\sim$ in the weaker sense that the two sides agree up to any desired order $I^{-K}$ with a well-defined remainder of lower order. The leading order term in the $*$-product is simply the product of the principal symbols.

The first step in constructing a Szegö projector $\Pi$ is to construct an approximate projector $S$, satisfying

$$
S^{2} \sim S \sim S^{*}, \quad S \in I^{0}(X \times X, C), \quad s_{0}(x, x)=\pi^{-m} \operatorname{det} L_{X}\|d \rho\| \quad(\bmod \psi) .
$$

The very first term of $s * s \sim s$ gives the equation ([BoSj], (4.10))

$$
s_{0}=\pi^{-m}\left(h_{\varphi}\right)^{-\frac{1}{2}} \bmod \psi
$$

where $h_{\varphi}$ is the (non-vanishing) Hessian determinant in $(\sigma, w)$ of the function $\varphi(x, y, w, \sigma)=$ $\psi(x, w)+\sigma \psi(w, y)$. On the diagonal $x=y$ one has $\left(h_{\varphi}\right)^{\frac{1}{2}}=\left(\operatorname{det} L_{X}\right)\|d \rho\|$.

We now observe that the amplitude of $S$ is not unique, since we may add to it any amplitude of the form $\left(D_{t}-\psi\right) a$ where $D_{t}=\frac{\partial}{i \partial t}$. This follows from the fact that $\left(D_{t}-\psi\right) e^{i t \psi}=$ 0 and from the fact that we can integrate by parts in an oscillatory integral. We note that $I\left(q+\left(D_{t}-\psi\right) a, \psi\right)=I(q, \psi)$ so the product descends to the quotient by the ideal $\left(D_{t}-\psi\right) S^{M}$. We abbreviate the statement $I\left(q+\left(D_{t}-\psi\right) a, \psi\right)=I(q, \psi)$ by $q \equiv q+\left(D_{t}-\psi\right) a$. Since we may absorb the terms $\psi\left(s_{0}-\pi^{-m}\left(h_{\varphi}\right)^{-\frac{1}{2}}\right)$ into $t^{m-1} s_{1}$, the difference being of the form $\left(D_{t}-\psi\right) a$, we may take $s_{0}$ to be defined by (48).

We now apply an argument given in [BoSj] to determine the rest of the amplitude $s$ from $s_{0}$ and by the equations $s^{*}=s \sim s * s$. Indeed, since $S^{2} \sim S \sim S^{*}$, there exists $r \in S^{-1}\left(X \times X \times \mathbb{R}^{+}\right)$with

$$
s_{0} * s_{0}=s_{0}+r
$$

Clearly, $r * s_{0}=s_{0} * r$. We now construct $E(r)$ so that

$$
s=s_{0}+E(r) \Longrightarrow s * s \sim s, s^{*}=s .
$$

Omitting the $*$ since the product is commutative, we get:

$$
\begin{aligned}
& \left(2 s_{0}-1\right) E(r)+E(r)^{2}=-r \Longrightarrow 2 \sqrt{r+\frac{1}{4}} E(r)+E(r)^{2}=-r \\
& \Longrightarrow E(r) \sim \frac{1}{2}-\sqrt{r+\frac{1}{4}} .
\end{aligned}
$$

The right hand side is a formal power series in $*$ products of $r$. Given a prescribed symbol order in advance, we may truncate the $*$ product to obtain a convergent expansion for $E(r)$ plus a remainder which is lower than the prescribed order. The resulting amplitude $s$ will satisfy $s^{*}=s \sim s * s$ to the prescribed order and hence be an amplitude for an approximate Szegö projector $S$. Thus, for any $K$ we can construct an approximate Szegö projector $S_{K}$ such that $S_{K}^{2}=S_{K}+E_{K}=S_{K}^{*}+E_{K}^{*}$ where $E_{K}(x, y)$ lies in $\mathcal{C}^{K}(X \times X)$. As discussed in [BoSi], one can in fact produce an approximate projector modulo smoothing operators.

We then modify $S$ by a smoothing operator $E$ to obtain a true projection (see BoGu, Appendix A.4): Since $S^{2} \sim S$ and $S=S^{*}$, it follows that the spectrum of $S$ is concentrated near $\{0,1\}$. Let $U_{0}, U_{1}$ be disjoint open sets containing the points of the spectrum near 0,1 , respectively, and let $F$ be the analytic (locally constant) function on $U_{0} \cup U_{1}$ given by $F\left(U_{0}\right)=\{0\}, F\left(U_{1}\right)=\{1\}$. Hence $F(S):=\Pi$ is a true projection. More concretely, since $S$ is self-adjoint it has an eigenfunction expansion $S(x, y)=\sum_{n=1}^{\infty} \lambda_{n} f_{n}(x) f_{n}(y)$. We may collect (modulo a finite dimensional ambiguity) the eigenvalues which cluster at $\{0\}$ and those 
which cluster at $\{1\}$ to obtain $S(x, y)=\sum_{n: \lambda_{n} \in U_{0}} \lambda_{n} f_{n}(x) f_{n}(y)+\sum_{n: \lambda_{n} \in U_{1}} \lambda_{n} f_{n}(x) f_{n}(y)$. Then $F(S)(x, y)=\sum_{n: \lambda_{n} \in U_{1}} f_{n}(x) f_{n}(y)$.

To summarize the above discussion, a Szegö projector $\Pi$ can be written in the form:

$$
\begin{gathered}
\Pi(x, y)=S(x, y)+E(x, y), \\
S(x, y)=\int_{0}^{\infty} e^{i t \psi(x, y)} s(x, y, t) d t, \quad E(x, y) \in \mathcal{C}^{\infty}(X \times X),
\end{gathered}
$$

where $\psi$ is given by Theorem 2.3 and $s \sim \sum_{k=0}^{\infty} t^{m-k} s_{k}(x, y) \in S^{m}\left(X \times X \times \mathbb{R}^{+}\right)$is constructed as above. Although $\Pi$ is not unique, the above construction defines a canonical choice of $\Pi$ modulo smoothing operators. In the complex case, the construction produces the usual Szegö projector $\Pi$ onto the kernel of $\bar{\partial}_{b}$, and (52) is the Boutet de Monvel-Sjöstrand oscillatory integral formula for it ([BoSj, Th. 1.5 and $\S 2 . c])$.

2.5. Almost holomorphic sections. In the complex case, a holomorphic section $s$ of $L^{N}$ lifts to a $\hat{s} \in \mathcal{L}_{N}^{2}(X)$ which satisfying $\bar{\partial}_{b} \hat{s}=0$. The operator $\bar{\partial}_{b}$ extends to a complex satisfying $\bar{\partial}_{b}^{2}=0$, which is a necessary and sufficient condition for having a maximal family of CR holomorphic coordinates. In the non-integrable case $\bar{\partial}_{b}^{2} \neq 0$, and there may be no local solutions of $\bar{\partial}_{b} f=0$. We now quote a result of Boutet-de-Monvel and Guillemin which replaces $\bar{\partial}_{b}$ with a pseudodifferential operator $\bar{D}_{0}$ so that $\bar{D}_{0} \Pi=0$. Indeed, Boutet de Monvel Bout and Boutet de Monvel - Guillemin BoGu defined a complex $\bar{D}_{j}$, which is a good replacement for $\bar{\partial}_{b}$ in the non-integrable case. Their main result is:

TheOREM 2.5. (see [BoGu], Lemma 14.11 and Theorem A 5.9) There exists an $S^{1}$-invariant complex of first order pseudodifferential operators $\bar{D}_{j}$ over $X$

$$
0 \rightarrow \mathcal{C}^{\infty}\left(\Lambda_{b}^{0,0}\right) \stackrel{\bar{D}_{0}}{\rightarrow} \mathcal{C}^{\infty}\left(\Lambda_{b}^{0,1}\right) \stackrel{\bar{D}_{1}}{\rightarrow} \cdots \stackrel{\bar{D}_{m-1}}{\longrightarrow} \mathcal{C}^{\infty}\left(\Lambda_{b}^{0, m}\right) \rightarrow 0
$$

where $\Lambda_{b}^{0, j}=\Lambda^{j}\left(H^{0,1} X\right)^{*}$, such that:

i) $\sigma\left(\bar{D}_{j}\right)=\sigma\left(\bar{\partial}_{b}\right)$ to second order along $\Sigma:=\left\{\left(x, r \alpha_{x}\right): x \in X, r>0\right\} \subset T^{*} X$;

ii) The Szegö kernel $\Pi$ is the orthogonal projector onto the kernel of $\bar{D}_{0}$;

iii) $\left(\bar{D}_{0}, \frac{\partial}{\partial \theta}\right)$ is jointly elliptic.

Let us briefly summarize the construction of $\bar{D}_{0}$ (following [BoGu, Appendix]). We begin with any $S^{1}$-equivariant symmetric first order pseudodifferential operator $\bar{D}_{0}^{\prime}$ with principal symbol equal to $\sum_{j=1}^{m} \zeta_{j} \bar{\vartheta}_{j}$. Then $\bar{D}_{0}^{\prime} \Pi$ is of order $\leq 0$ so one may find a zeroth order pseudodifferential operator $Q_{0}$ such that $\bar{D}_{0}^{\prime} \Pi \sim Q_{0} \Pi$ (modulo smoothing operators). Then put: $\bar{D}_{0}=\left(\bar{D}_{0}^{\prime}-Q_{0}\right)-\left(\bar{D}_{0}^{\prime}-Q_{0}\right) \Pi$. Clearly, $\bar{D}_{0} \Pi=0$ and $\sigma\left(\bar{D}_{0}\right)=\sigma\left(\bar{D}_{0}^{\prime}\right)=\sum_{j=1}^{m} \zeta_{j} \bar{\vartheta}_{j}$. The characteristic variety of $\bar{D}_{0}$ is then equal to $\Sigma$. Since $p_{\theta}$ is the symbol of $\frac{\partial}{\partial \theta}$ and since the system $\left\{\sigma_{\bar{D}_{0}}, p_{\theta}\right\}$ has no zeros in $T^{*} X-0$ it follows that $\left\{\bar{D}_{0}, \frac{\partial}{\partial \theta}\right\}$ is an elliptic system.

One can then construct the higher $\bar{D}_{j}$ recursively so that $\bar{D}_{j} \bar{D}_{j-1}=0$. We refer to BoGu, Appendix $\S 5$, for further details.

We refer to the kernel $\mathcal{H}^{2}(X)=\operatorname{ker} \bar{D}_{0} \cap \mathcal{L}^{2}(X)$ as the Hardy space of square-integrable 'almost CR functions' on $X$. The $\mathcal{L}^{2}$ norm is with respect to the inner product

$$
\left\langle F_{1}, F_{2}\right\rangle=\frac{1}{2 \pi} \int_{X} F_{1} \overline{F_{2}} d V_{X}, \quad F_{1}, F_{2} \in \mathcal{L}^{2}(X),
$$


where

$$
d V_{X}=\frac{1}{m !} \alpha \wedge(d \alpha)^{m}=\alpha \wedge \pi^{*} d V_{M}
$$

The $S^{1}$ action on $X$ commutes with $\bar{D}_{0}$; hence $\mathcal{H}^{2}(X)=\bigoplus_{N=0}^{\infty} \mathcal{H}_{N}^{2}(X)$ where $\mathcal{H}_{N}^{2}(X)=$ $\left\{F \in \mathcal{H}^{2}(X): F\left(r_{\theta} x\right)=e^{i N \theta} F(x)\right\}$. We denote by $H_{J}^{0}\left(M, L^{N}\right)$ the space of sections which corresponds to $\mathcal{H}_{N}^{2}(X)$ under the map $s \mapsto \hat{s}$. Elements of $H_{J}^{0}\left(M, L^{N}\right)$ are the almost holomorphic sections of $L^{N}$. (Note that products of almost holomorphic sections are not necessarily almost holomorphic.) We henceforth write $\hat{s}=s$ and identify $H_{J}^{0}\left(M, L^{N}\right)$ with $\mathcal{H}_{N}^{2}(X)$. Since $\left(\bar{D}_{0}, \frac{\partial}{\partial \theta}\right)$ is a jointly elliptic system, elements of $H_{J}^{0}\left(M, L^{N}\right)$ and $\mathcal{H}_{N}^{2}(X)$ are smooth. In many other respects, $H_{J}^{0}\left(M, L^{N}\right)$ is analogous to the space of holomorphic sections in the complex case. Subsequent results will bear this out.

We let $\Pi_{N}: \mathcal{L}^{2}(X) \rightarrow \mathcal{H}_{N}^{2}(X)$ denote the orthogonal projection. The level $N$ Szegö kernel $\Pi_{N}(x, y)$ is defined by

$$
\Pi_{N} F(x)=\int_{X} \Pi_{N}(x, y) F(y) d V_{X}(y), \quad F \in \mathcal{L}^{2}(X) .
$$

It can be given as

$$
\Pi_{N}(x, y)=\sum_{j=1}^{d_{N}} S_{j}^{N}(x) \overline{S_{j}^{N}(y)},
$$

where $S_{1}^{N}, \ldots, S_{d_{N}}^{N}$ form an orthonormal basis of $\mathcal{H}_{N}^{2}(X)$.

Remark: The results stated here use only the $\bar{D}_{0}$ term of the complex; its kernel consists of the spaces of almost holomorphic sections of the powers $L^{N}$ of the line bundle $L$, as explained below. The complex $\bar{D}_{j}$ was used by Boutet de Monvel -Guillemin [BoGu, Lemma 14.14] to show that the dimension of $H_{J}^{0}\left(M, L^{N}\right)$ or $\mathcal{H}_{N}^{2}(X)$ is given by the Riemann-Roch formula (for $N$ sufficiently large). For our results, we need only the leading term of Riemann-Roch, which we obtain as a consequence of Theorem 2 (a). (The reader should be warned that the symbol is described incorrectly in Lemma 14.11 of $B$ BoGu. However, it is correctly described in Theorem 5.9 of the Appendix to [BoGu] and also in [GuOr]).

\section{SCALing ASYmptotics For SzegÖ KeRnels}

In [BSZ1, Theorem 3.1], we showed that in the complex case, the scaled Szegö kernel $\Pi_{N}$ near the diagonal is asymptotic to the Szegö kernel $\Pi_{1}^{\mathbf{H}}$ of level one for the reduced Heisenberg group, given by

$$
\Pi_{1}^{\mathbf{H}}(z, \theta ; w, \varphi)=\frac{1}{\pi^{m}} e^{i(\theta-\varphi)+i \Im(z \cdot \bar{w})-\frac{1}{2}|z-w|^{2}}=\frac{1}{\pi^{m}} e^{i(\theta-\varphi)+z \cdot \bar{w}-\frac{1}{2}\left(|z|^{2}+|w|^{2}\right)} .
$$

The method was to apply the Boutet de Monvel-Sjöstrand oscillatory integral formula

$$
\Pi(x, y) \sim \int_{0}^{\infty} e^{i t \psi(x, y)} s(x, y, t) d t \bmod \mathcal{C}^{\infty}(X \times X)
$$

arising from a parametrix construction (recall (52)).

Our goal now is to show that the universal asymptotic formula of BSZ1 for the neardiagonal scaled Szegö kernel holds for the symplectic case (Theorem [3.1). In fact, our description adds some quite useful details to the formula given in [BSZ1]. 
We have shown that Szegö kernels can be expressed in the form $\Pi(x, y)=S(x, y)+E(x, y)$, where $S$ is the Fourier integral kernel in (52) and where $E \in \mathcal{C}^{\infty}(X \times X)$ is the remainder. We denote the $N^{\text {th }}$ Fourier coefficient of these operators relative to the $S^{1}$ action by $\Pi_{N}=$ $S_{N}+E_{N}$. Since $E$ is smooth, we have $E_{N}(x, y)=O\left(N^{-\infty}\right)$, where $O\left(N^{-\infty}\right)$ denotes a quantity which is uniformly $O\left(N^{-k}\right)$ on $X \times X$ for all positive $k$. The Fourier coefficients $S_{N}$ are given by

$$
S_{N}(x, y)=\int_{0}^{2 \pi} e^{-i N \theta} S\left(r_{\theta} x, y\right) d \theta=\int_{0}^{\infty} \int_{0}^{2 \pi} e^{-i N \theta} e^{i t \psi\left(r_{\theta} x, y\right)} s\left(r_{\theta} x, y, t\right) d \theta d t
$$

where $r_{\theta}$ denotes the $S^{1}$ action on $X$. Changing variables $t \mapsto N t$ gives

$$
S_{N}(x, y)=N \int_{0}^{\infty} \int_{0}^{2 \pi} e^{i N\left(-\theta+t \psi\left(r_{\theta} x, y\right)\right)} s\left(r_{\theta} x, y, N t\right) d \theta d t .
$$

We now determine the scaling limit of the Szegö kernel by continuing the argument of [BSZ1], and adding some new details on homogeneities which will be useful in our applications. To describe the scaling limit at a point $x_{0} \in X$, we choose a Heisenberg chart $\rho: U, 0 \rightarrow X, x_{0}$ centered at $P_{0}=\pi\left(x_{0}\right) \in M$. Recall (\$1.2) that choosing $\rho$ is equivalent to choosing preferred coordinates centered at $P_{0}$ and a preferred local frame $e_{L}$ at $P_{0}$. We then write the Szegö kernel $\Pi_{N}$ in terms of these coordinates:

$$
\Pi_{N}^{P_{0}}(u, \theta ; v, \varphi)=\Pi_{N}(\rho(u, \theta), \rho(v, \varphi)),
$$

where the superscript $P_{0}$ is a reminder that we are using coordinates centered at $P_{0}$. (We remark that the function $\Pi_{N}^{P_{0}}$ depends also on the choice of preferred coordinates and preferred frame, which we omit from the notation.) The first term in our asymptotic formula below says that the $N^{\text {th }}$ scaled Szegö kernel looks approximately like the Szegö kernel of level one for the reduced Heisenberg group (recall (57)):

$$
\Pi_{N}^{P_{0}}\left(\frac{u}{\sqrt{N}}, \frac{\theta}{N} ; \frac{v}{\sqrt{N}}, \frac{\varphi}{N}\right) \approx \Pi_{1}^{\mathbf{H}}(u, \theta ; v, \varphi)=\frac{1}{\pi^{m}} e^{i(\theta-\varphi)+i \Im(u \cdot \bar{v})-\frac{1}{2}|u-v|^{2}} .
$$

In the following, we shall denote the Taylor series of a $\mathcal{C}^{\infty}$ function $f$ defined in a neighborhood of $0 \in \mathbb{R}^{K}$ by $f \sim f_{0}+f_{1}+f_{2}+\ldots$ where $f_{j}$ is the homogeneous polynomial part of degree $j$. We also denote by $R_{n}^{f} \sim f_{n+1}+\cdots$ the remainder term in the Taylor expansion.

The following is our main result on the scaling asymptotics of the Szegö kernels near the diagonal. Since the result is of independent interest, we state our asymptotic formula in a more precise form than is needed for the applications in this paper.

Theorem 3.1. Let $P_{0} \in M$ and choose a Heisenberg coordinate chart about $P_{0}$. Then

$$
\begin{aligned}
N^{-m} & \Pi_{N}^{P_{0}}\left(\frac{u}{\sqrt{N}}, \frac{\theta}{N} ; \frac{v}{\sqrt{N}}, \frac{\varphi}{N}\right) \\
& =\Pi_{1}^{\mathbf{H}}(u, \theta ; v, \varphi)\left[1+\sum_{r=1}^{K} N^{-r / 2} b_{r}\left(P_{0}, u, v\right)+N^{-(K+1) / 2} R_{K}\left(P_{0}, u, v, N\right)\right],
\end{aligned}
$$

where:

- $b_{r}=\sum_{\alpha=0}^{2[r / 2]} \sum_{j=0}^{[3 r / 2]}\left(\psi_{2}\right)^{\alpha} Q_{r, \alpha, 3 r-2 j}$, where $Q_{r, \alpha, d}$ is homogeneous of degree $d$ and

$$
\psi_{2}(u, v)=u \cdot \bar{v}-\frac{1}{2}\left(|u|^{2}+|v|^{2}\right)
$$


in particular, $b_{r}$ has only even homogeneity if $r$ is even, and only odd homogeneity if $r$ is odd;

- $\left\|R_{K}\left(P_{0}, u, v, N\right)\right\|_{\mathcal{C}^{j}(\{|u| \leq \rho,|v| \leq \rho\}} \leq C_{K, j, \rho}$ for $j \geq 0, \rho>0$ and $C_{K, j, \rho}$ is independent of the point $P_{0}$ and choice of coordinates.

Proof. Since $\Pi_{N}=S_{N}+O\left(N^{-\infty}\right)$, it suffices to give the asymptotics of $S_{N}$. Hence, we fix $P_{0}$ and consider the asymptotics of

$$
\begin{aligned}
& S_{N}^{P_{0}}\left(\frac{u}{\sqrt{N}}, 0 ; \frac{v}{\sqrt{N}}, 0\right) \\
& \quad=N \int_{0}^{\infty} \int_{0}^{2 \pi} e^{i N\left(-\theta+t \psi\left(\frac{u}{\sqrt{N}}, \theta ; \frac{v}{\sqrt{N}}, 0\right)\right)} s\left(\frac{u}{\sqrt{N}}, \theta ; \frac{v}{\sqrt{N}}, 0, N t\right) d \theta d t
\end{aligned}
$$

where $\psi$ and $s$ are the phase and symbol from (52) written in terms of the Heisenberg coordinates. 2.3 ,

On $X$ we have $\lambda=a(z)^{-\frac{1}{2}} e^{i \varphi}$. So for $(x, y)=\left(z, \varphi, w, \varphi^{\prime}\right) \in X \times X$, we have by Theorem

$$
\psi\left(z, \varphi, w, \varphi^{\prime}\right)=i\left[1-\frac{a(z, w)}{\sqrt{a(z)} \sqrt{a(w)}} e^{i\left(\varphi-\varphi^{\prime}\right)}\right] .
$$

It follows that

$$
\begin{aligned}
& \psi\left(\frac{u}{\sqrt{N}}, \theta ; \frac{v}{\sqrt{N}}, 0\right) \\
& \quad=i\left[1-\frac{a\left(\frac{u}{\sqrt{N}}, \frac{v}{\sqrt{N}}\right)}{\sqrt{a\left(\frac{u}{\sqrt{N}}, \frac{u}{\sqrt{N}}\right)} \sqrt{a\left(\frac{v}{\sqrt{N}}, \frac{v}{\sqrt{N}}\right)}} e^{i \theta}\right] .
\end{aligned}
$$

We observe that the asymptotic expansion of a function $f\left(\frac{u}{\sqrt{N}}, \frac{v}{\sqrt{N}}\right)$ in powers of $N^{-\frac{1}{2}}$ is just the Taylor expansion of $f$ at $u=v=0$. By Lemma 2.4 and the notational convention established above, we have

$$
a\left(\frac{u}{\sqrt{N}}, \frac{v}{\sqrt{N}}\right)=1+\frac{1}{N} u \cdot \bar{v}+R_{3}^{a}\left(\frac{u}{\sqrt{N}}, \frac{v}{\sqrt{N}}\right), \quad R_{3}^{a}\left(\frac{u}{\sqrt{N}}, \frac{v}{\sqrt{N}}\right)=O\left(N^{-3 / 2}\right) .
$$

The phase in (60)

$$
\widetilde{\Psi}:=t \psi\left(\frac{u}{\sqrt{N}}, \theta ; \frac{v}{\sqrt{N}}, 0\right)-\theta=i t\left[1-\frac{a\left(\frac{u}{\sqrt{N}}, \frac{v}{\sqrt{N}}\right)}{a\left(\frac{u}{\sqrt{N}}, \frac{u}{\sqrt{N}}\right)^{\frac{1}{2}} a\left(\frac{v}{\sqrt{N}}, \frac{v}{\sqrt{N}}\right)^{\frac{1}{2}}} e^{i \theta}\right]-\theta
$$

then has the asymptotic $N$-expansion

$$
\widetilde{\Psi}=i t\left[1-e^{i \theta}\right]-\theta-\frac{i t}{N} \psi_{2}(u, v) e^{i \theta}+t R_{3}^{\psi}\left(\frac{u}{\sqrt{N}}, \frac{v}{\sqrt{N}}\right) e^{i \theta} .
$$


We use a smooth partition of unity $\left\{\rho_{1}(t), \rho_{2}(t)\right\}$ to decompose the integral (60) into one over $0<t<3$ and one over $t>2$ :

$$
\begin{aligned}
S_{N}^{P_{0}}\left(\frac{u}{\sqrt{N}}, 0 ; \frac{v}{\sqrt{N}}, 0\right) & =I_{1}+I_{2} \\
I_{1} & =N \int_{0}^{3} \int_{0}^{2 \pi} e^{i N \widetilde{\Psi}} \rho_{1}(t) s\left(\frac{u}{\sqrt{N}}, \theta ; \frac{v}{\sqrt{N}}, 0, N t\right) d \theta d t \\
I_{2} & =N \int_{2}^{\infty} \int_{0}^{2 \pi} e^{i N \widetilde{\Psi}} \rho_{2}(t) s\left(\frac{u}{\sqrt{N}}, \theta ; \frac{v}{\sqrt{N}}, 0, N t\right) d \theta d t .
\end{aligned}
$$

To evaluate $I_{1}$, we absorb $\left(-i \psi_{2}+N R_{3}^{\psi}\right) t e^{i \theta}$ into the amplitude (as in [BSZ1]), so that we view $I_{1}$ as an oscillatory integral with phase

$$
\Psi(t, \theta):=i t\left(1-e^{i \theta}\right)-\theta
$$

and with amplitude

$$
A\left(t, \theta ; P_{0}, u, v\right):=\rho_{1}(t) e^{t e^{i \theta} \psi_{2}(u, v)+i t e^{i \theta} N R_{3}^{\psi}\left(\frac{u}{\sqrt{N}}, \frac{v}{\sqrt{N}}\right)} s\left(\frac{u}{\sqrt{N}}, \theta ; \frac{v}{\sqrt{N}}, 0, N t\right) ;
$$

i.e.,

$$
I_{1}=N \int_{0}^{3} \int_{0}^{2 \pi} e^{i N \Psi(t, \theta)} A\left(t, \theta ; P_{0}, u, v\right) d \theta d t
$$

We evaluate (70) by the method of stationary phase as in BSZ1. The phase $\Psi$ is independent of the parameters $(u, v)$ and we have

$$
\begin{aligned}
& \frac{\partial}{\partial t} \Psi=i\left(1-e^{i \theta}\right) \\
& \frac{\partial}{\partial \theta} \Psi=t e^{i \theta}-1
\end{aligned}
$$

so the critical set of the phase is the point $\{t=1, \theta=0\}$. The Hessian $\Psi^{\prime \prime}$ on the critical set equals

$$
\left(\begin{array}{ll}
0 & 1 \\
1 & i
\end{array}\right)
$$

so the phase is non-degenerate and the Hessian operator $L_{\Psi}$ is given by

$$
L_{\Psi}=\left\langle\Psi^{\prime \prime}(1,0)^{-1} D, D\right\rangle=2 \frac{\partial^{2}}{\partial t \partial \theta}-i \frac{\partial^{2}}{\partial t^{2}} .
$$

By the stationary phase method for complex oscillatory integrals ([Hör], Theorem 7.7.5), we have

$$
I_{1}=\left.\gamma \sum_{j=0}^{J} N^{-j} L_{j}\left[A\left(t, \theta ; P_{0}, u, v\right)\right]\right|_{t=1, \theta=0}+\widehat{R}_{J}\left(P_{0}, u, v, N\right),
$$

where

$$
\gamma=N \frac{1}{\sqrt{\operatorname{det}\left(\mathrm{N \Psi}^{\prime \prime}(1,0) / 2 \pi \mathrm{i}\right)}}=\sqrt{-2 \pi i}
$$

and $L_{j}$ is the differential operator of order $2 j$ in $(t, \theta)$ defined by

$$
L_{j} \varphi(t, \theta)=\sum_{\nu-\mu=j} \sum_{2 \nu \geq 3 \mu} \frac{1}{2^{\nu} i^{j} \mu ! \nu !} L_{\Psi}^{\nu}\left[\varphi(t, \theta)\left(R_{3}^{\Psi}\right)^{\mu}(t, \theta)\right]
$$

with $R_{3}^{\Psi}(t, \theta)$ the third order remainder in the Taylor expansion of $\Psi$ at $(t, \theta)=(1,0)$. 
Also, the remainder is estimated by

$$
\left|\widehat{R}_{J}\left(P_{0}, u, v, N\right)\right| \leq C N^{-J} \sum_{|\alpha| \leq 2 J+2} \sup _{t, \theta}\left|D_{t, \theta}^{\alpha} A\left(t, \theta ; P_{0}, u, v\right)\right| \leq C_{J} N^{m-J},
$$

where the last inequality follows by observing from (69) that

$$
\left|A\left(t, \theta ; P_{0}, u, v\right)\right| \leq C^{\prime}\left|s\left(\frac{u}{\sqrt{N}}, \theta ; \frac{v}{\sqrt{N}}, 0, N t\right)\right| \leq C^{\prime \prime} N^{m} \quad(0 \leq t \leq 3),
$$

and similarly for its derivatives (using the fact that $s$ is a symbol of order $m$ and hence $D_{t, \theta}^{\alpha} s\left(r_{\theta} x, y, N t\right)=O\left(N^{m}\right)$ uniformly for $\left.t \leq 3\right)$.

To evaluate (72), we first expand $\exp \left[i t e^{i \theta} N R_{3}^{\psi}\left(\frac{u}{\sqrt{N}}, \frac{v}{\sqrt{N}}\right)\right]$ in powers of $N^{-\frac{1}{2}}$, keeping track of the homogeneity in $(u, v)$ of the coefficients. We simplify the notation by writing $g=t e^{i \theta}$. By definition,

$$
R_{3}^{\psi}\left(\frac{u}{\sqrt{N}}, \frac{v}{\sqrt{N}}\right) \sim N^{-3 / 2} \psi_{3}(u, v)+N^{-2} \psi_{4}(u, v)+\cdots+N^{-d / 2} \psi_{d}(u, v)+\cdots .
$$

We then have

$$
e^{i N g R_{3}^{\psi}\left(\frac{u}{\sqrt{N}}, \frac{v}{\sqrt{N}}\right)} \sim \sum_{r=0}^{\infty} N^{-r / 2} c_{r}(u, v ; t, \theta) .
$$

We further expand

$$
c_{r}=\sum_{\lambda=1}^{r} c_{r, r+2 \lambda}(u, v ; t, \theta), r \geq 1, \quad c_{0}=c_{00}=1,
$$

with $c_{r d}$ homogeneous of degree $d$ in $u, v$. Note that $c_{r}$ is a polynomial of degree $r$ in $g$. (The explicit formula for $c_{r d}$ is:

$$
c_{r d}=\sum\left\{\frac{1}{n !}(i g)^{n} \Pi_{j=1}^{n} \psi_{a_{j}}(u, v): n \geq 1, a_{j} \geq 3, \sum_{j=1}^{n} a_{j}=d, \sum_{j=1}^{n}\left(a_{j}-2\right)=r\right\}, r \geq 1 .
$$

The range of $d$ is determined by the fact that $d=\sum_{j=1}^{n} a_{j}=r+2 n$ with $0 \leq n \leq r$.)

We similarly expand the symbol:

$$
s\left(\frac{u}{\sqrt{N}}, \theta ; \frac{v}{\sqrt{N}}, 0, N t\right)=\sum_{k=0}^{\infty} N^{m-k} t^{m-k} s_{k}\left(\frac{u}{\sqrt{N}}, \frac{v}{\sqrt{N}}, \theta\right)=\sum_{k, \ell=0}^{\infty} N^{m-k-\ell / 2} t^{m-k} s_{k \ell}(u, v, \theta)
$$

where $s_{k \ell}$ is the homogeneous term of $s_{k}$ of degree $\ell$ in $(u, v)$. Hence, we have

$$
\begin{aligned}
A & \sim \rho_{1}(t) e^{g \psi_{2}(u, v)+i N g R_{3}^{\psi}\left(\frac{u}{\sqrt{N}}, \frac{v}{\sqrt{N}}, \theta\right)} \sum_{k=0}^{\infty} N^{m-k} t^{m-k} s_{k}\left(\frac{u}{\sqrt{N}}, \frac{v}{\sqrt{N}}, \theta\right) \\
& \sim \rho_{1}(t) e^{g \psi_{2}(u, v)} N^{m} \sum_{n=0}^{\infty} N^{-n / 2} f_{n}\left(u, v ; t, \theta, P_{0}\right),
\end{aligned}
$$

where the remainder in (88) after summing $K$ terms is $O\left(N^{m-\frac{K+1}{2}}\right)$. (Note that $f_{n}$ is a polynomial in $t$ of degree $n+m$.) We further have

$$
f_{n}=\sum_{r+\ell+2 k=n} c_{r} s_{k \ell}=\sum_{k=0}^{[n / 2]} t^{m-k}\left(s_{k, n-2 k}+\sum_{r=1}^{n-2 k} \sum_{\lambda=1}^{r} c_{r, r+2 \lambda} s_{k, n-2 k-r}\right)=\sum_{j=0}^{[3 n / 2]} f_{n, 3 n-2 j},
$$


where $f_{n, d}$ is homogeneous of degree $d$ in $(u, v)$.

Hence,

$$
\left.L_{j}[A]\right|_{t=1, \theta=0}=\left.\sum_{n=0}^{K} N^{m-n / 2} L_{j}\left[e^{g \psi_{2}} f_{n}\right]\right|_{t=1, \theta=0}+O\left(N^{m-\frac{K+1}{2}}\right) .
$$

Since $L_{\Psi}$ is a second order operator in $(t, \theta)$, we see that

$$
\left.L_{j}\left[e^{g \psi_{2}} f_{n}\right]\right|_{t=1, \theta=0}=e^{\psi_{2}} \sum_{\alpha \leq 2 j}\left(\psi_{2}\right)^{\alpha} F_{n j \alpha},
$$

where the $F_{n j \alpha}$ are polynomials in $u, v, \bar{u}, \bar{v}$ of degree $\leq 3 n$. Therefore, by (72), (79) and (80), we have

$$
\begin{aligned}
N^{-m} I_{1} & \sim \sqrt{-2 \pi i} e^{\psi_{2}} \sum_{n=0}^{\infty} \sum_{j=0}^{\infty} \sum_{\alpha=0}^{2 j}\left(\psi_{2}\right)^{\alpha} N^{-\frac{n}{2}-j} F_{n j \alpha} \\
& \sim \sqrt{-2 \pi i} e^{\psi_{2}} \sum_{r=0}^{\infty} \sum_{j=0}^{[r / 2]} \sum_{\alpha=0}^{2 j}\left(\psi_{2}\right)^{\alpha} N^{-r / 2} F_{r-2 j, j, \alpha} \\
& \sim e^{\psi_{2}} \sum_{r=0}^{\infty} \sum_{\alpha=0}^{2[r / 2]}\left(\psi_{2}\right)^{\alpha} N^{-r / 2} Q_{r \alpha} .
\end{aligned}
$$

As with $f_{n}$ we have the homogeneous expansion:

$$
Q_{r \alpha}=\sum_{j=0}^{[3 r / 2]} Q_{r, \alpha, 3 r-2 j}
$$

Here, $Q_{r, \alpha, d}$ is homogeneous of degree $d$ in $(u, v)$. Thus we have the desired Taylor series for $I_{1}$.

To show that $I_{2}=O\left(N^{-\infty}\right)$, we observe from (65) that

$$
\left|\frac{\partial}{\partial \theta} \widetilde{\Psi}\right| \geq\left[1-O\left(N^{-1}\right)\right] t-1 \geq \frac{1}{3} t \quad \text { for } t \geq 2, N \gg 1 .
$$

Hence by [Hör], Theorem 7.7.1, for all integers $k \geq 1$ we have

$$
\begin{aligned}
\int_{0}^{2 \pi} e^{i N \widetilde{\Psi}} \rho_{2}(t) s\left(\frac{u}{\sqrt{N}}, \theta ;\right. & \left.\frac{v}{\sqrt{N}}, 0, N t\right) d \theta \\
& \leq C^{\prime} N^{-k} t^{-k} \sum_{\alpha \leq k} \sup \left|D_{\theta}^{\alpha}\left(\rho_{2}(t) s\left(\frac{u}{\sqrt{N}}, \theta ; \frac{v}{\sqrt{N}}, 0, N t\right)\right)\right| \\
& \leq C_{k}^{\prime} N^{m-k} t^{m-k} .
\end{aligned}
$$

(To see that $C^{\prime}$ is independent of $t$, we write $i N \widetilde{\Psi}=i(N t) \Phi$, with $\Phi=\widetilde{\Psi} / t$ and apply [Hör, Theorem 7.7.1] to the phase $\Phi$.) Integrating over $t$, we conclude that $I_{2}=O\left(N^{m+1-k}\right)$; since $k \geq 1$ is arbitrary, $I_{2}=O\left(N^{-\infty}\right)$. The estimate for the remainder now follows from (74) and $(79)$. 


\section{Kodaira embedding and Tian almost isometry theorem}

Definition: By the Kodaira maps we mean the maps $\Phi_{N}: M \rightarrow P H^{0}\left(M, L^{N}\right)^{\prime}$ defined by $\Phi_{N}(z)=\left\{s^{N}: s^{N}(z)=0\right\}$. Equivalently, we can choose an orthonormal basis $S_{1}^{N}, \ldots, S_{d_{N}}^{N}$ of $H^{0}\left(M, L^{N}\right)$ and write

$$
\Phi_{N}: M \rightarrow \mathbb{C P}^{d_{N}-1}, \quad \Phi_{N}(z)=\left(S_{1}^{N}(z): \cdots: S_{d_{N}}^{N}(z)\right) .
$$

We also define the lifts of the Kodaira maps:

$$
\widetilde{\Phi}_{N}: X \rightarrow \mathbb{C}^{d_{N}}, \quad \widetilde{\Phi}_{N}(x)=\left(S_{1}^{N}(x), \ldots, S_{d_{N}}^{N}(x)\right) .
$$

Note that

in particular,

$$
\Pi_{N}(x, y)=\widetilde{\Phi}_{N}(x) \cdot \overline{\widetilde{\Phi}_{N}(y)}
$$

$$
\Pi_{N}(x, x)=\left\|\widetilde{\Phi}_{N}(x)\right\|^{2} .
$$

We now prove Theorem 2, which generalizes to the symplectic category the asymptotic expansion theorem of [Zel] (also proved independently by [Cat] using the Bergman kernel in place of the Szegö kernel) and Tian's approximate isometry theorem [Tian]:

(a) Using the expansion of Theorem 3.1 with $u=v=0$ and noting that $b_{r}(z, 0,0)=0$ for $r$ odd, we obtain the above expansion of $\Pi_{N}(z, 0 ; z, 0)$ with $a_{r}(z)=b_{2 r}(z, 0,0)$. (The expansion also follows by precisely the same proof as in Zell.)

(b) In the holomorphic case, (b) followed by differentiating (a), using that $\Phi_{N}^{*}\left(\partial \bar{\partial} \log |\xi|^{2}\right)=$ $\partial \bar{\partial} \log \left|\Phi_{N}\right|^{2}$. In the almost complex case, $\Phi_{N}^{*}$ does not commute with the complex derivatives, so we need to modify the proof. To do so, we use the following notation: the exterior derivative on a product manifold $Y_{1} \times Y_{2}$ can be decomposed as $d=d^{1}+d^{2}$, where $d^{1}$ and $d^{2}$ denote exterior differentiation on the first and second factors, respectively. (This is formally analogous to the decomposition $d=\partial+\bar{\partial}$; e.g., $d^{1} d^{1}=d^{2} d^{2}=d^{1} d^{2}+d^{2} d^{1}=0$.)

Recall that the Fubini-Study form $\omega_{F S}$ on $\mathbb{C P}^{m-1}$ is induced by the 2-form $\widetilde{\omega}_{m}=\frac{i}{2} \partial \bar{\partial} \log |\xi|^{2}$ on $\mathbb{C}^{m} \backslash\{0\}$. We consider the 2 -form $\Omega$ on $\left(\mathbb{C}^{m} \backslash\{0\}\right) \times\left(\mathbb{C}^{m} \backslash\{0\}\right)$ given by

$$
\Omega=\frac{i}{2} \partial \bar{\partial} \log \zeta \cdot \bar{\eta}=\frac{i}{2} d^{1} d^{2} \log \zeta \cdot \bar{\eta} .
$$

Note that $\Omega$ is smooth on a neighborhood of the diagonal $\{\zeta=\eta\}$, and

$$
\left.\Omega\right|_{\zeta=\eta}=\widetilde{\omega}_{m}
$$

(where the restriction to $\{\zeta=\eta\}$ means the pull-back under the map $\zeta \mapsto(\zeta, \zeta)$ ).

It suffices to show that

$$
\frac{1}{N} \tilde{\Phi}_{N}^{*} \omega_{d_{N}} \rightarrow \pi^{*} \omega, \quad \pi: X \rightarrow M .
$$

To do this, we consider the maps

$$
\Psi_{N}=\widetilde{\Phi}_{N} \times \widetilde{\Phi}_{N}: X \times X \rightarrow \mathbb{C}^{d_{N}} \times \mathbb{C}^{d_{N}}, \quad \Psi_{N}(x, y)=\left(\widetilde{\Phi}_{N}(x), \widetilde{\Phi}_{N}(y)\right) .
$$

It is elementary to check that $\Psi_{N}^{*}$ commutes with $d^{1}$ and $d^{2}$. By (85)), we have

$$
\Psi_{N}^{*}(\log \zeta \cdot \bar{\eta})=(\log \zeta \cdot \bar{\eta}) \circ \Psi_{N}=\log \Pi_{N} .
$$


Therefore,

$$
\frac{1}{N} \tilde{\Psi}_{N}^{*} \Omega_{d_{N}}=\frac{i}{2 N} \Psi_{N}^{*} d^{1} d^{2} \log \zeta \cdot \bar{\eta}=\frac{i}{2 N} d^{1} d^{2} \Psi_{N}^{*} \log \zeta \cdot \bar{\eta}=\frac{i}{2 N} d^{1} d^{2} \log \Pi_{N} .
$$

Restricting (87) to the diagonal, we then have

$$
\frac{1}{N} \tilde{\Phi}_{N}^{*} \omega_{d_{N}}=\left.\frac{i}{2 N}\left(d^{1} d^{2} \log \Pi_{N}\right)\right|_{x=y}=\operatorname{diag}^{*}\left(d^{1} d^{2} \log \Pi_{N}\right)
$$

where $\operatorname{diag}: X \rightarrow X \times X$ is the diagonal map $\operatorname{diag}(x)=(x, x)$.

Using Heisenberg coordinates as in Theorem 3.1, we have by the near-diagonal scaling asymptotics

$$
\begin{aligned}
\left.\frac{1}{N} \tilde{\Phi}_{N}^{*} \omega_{d_{N}}\right|_{P_{0}} & =\left.\frac{i}{2 N} \operatorname{diag}^{*} d^{1} d^{2} \log \Pi_{N}^{P_{0}}\left(\frac{u}{\sqrt{N}}, \frac{\theta}{N} ; \frac{v}{\sqrt{N}}, \frac{\varphi}{N}\right)\right|_{0} \\
& =\left.\frac{i}{2 N} \operatorname{diag}^{*} d^{1} d^{2} \log \Pi_{1}^{\mathbf{H}}(u, \theta ; v, \varphi)\right|_{0}+O\left(N^{-\frac{1}{2}}\right)
\end{aligned}
$$

Finally,

$$
\begin{aligned}
\left.\frac{i}{2 N} \operatorname{diag}^{*} d^{1} d^{2} \log \Pi_{1}^{\mathbf{H}}(u, \theta ; v, \varphi)\right|_{0} & =\frac{i}{2 N} \operatorname{diag}^{*} d^{1} d^{2}\left[i(\theta-\varphi)+u \cdot \bar{v}-\frac{1}{2}\left(|u|^{2}+|v|^{2}\right)\right] \\
& =\frac{i}{2 N} \sum_{q=1}^{m} d u_{q} \wedge d \bar{u}_{q}=\frac{i}{2} \sum_{q=1}^{m} d z_{q} \wedge d \bar{z}_{q}=\left.\omega\right|_{P_{0}} .
\end{aligned}
$$

Remark: A more explicit way to show (b) is to expand the Fubini-Study form:

$$
\widetilde{\omega}_{m}=\frac{i}{2}|\xi|^{-4}\left[|\xi|^{2} \sum_{j=1}^{m} d \xi_{j} \wedge d \bar{\xi}_{j}-\sum_{j, k=1}^{m} \bar{\xi}_{j} \xi_{k} d \xi_{j} \wedge d \bar{\xi}_{k}\right] .
$$

Then

$$
\frac{1}{N} \tilde{\Phi}_{N}^{*} \omega_{d_{N}}=\frac{i}{2} \Pi_{N}(x, x)^{-2}\left\{\left.\left(\Pi_{N}(x, x) d^{1} d^{2} \Pi_{N}(x, y)-d^{1} \Pi_{N}(x, y) \wedge d^{2} \Pi_{N}(x, y)\right\}\right|_{x=y}\right.
$$

and (b) follows from a short computation using Theorem 3.1 as above.

It follows from Theorem 2(b) that $\Phi_{N}$ is an immersion for $N \gg 0$. Using in part an idea of Bouche Bch, we now give a simple proof that the Kodaira embedding theorem holds for symplectic manifolds (Theorem B]):

Let $\left\{P_{N}, Q_{N}\right\}$ be any sequence of distinct points such that $\Phi_{N}\left(P_{N}\right)=\Phi_{N}\left(Q_{N}\right)$. By passing to a subsequence we may assume that one of the following two cases holds:

(i) The distance $r_{N}:=\operatorname{dist}\left(P_{N}, Q_{N}\right)$ between $P_{N}, Q_{N}$ satisfies $r_{N} \sqrt{N} \rightarrow \infty$;

(ii) There exists a constant $C$ independent of $N$ such that $r_{N} \leq C / \sqrt{N}$.

To prove that case (i) cannot occur, we let $\Pi_{N}^{P_{N}}(x)=\Pi_{N}\left(x, P_{N}\right)$ denote the 'peak section' at $P_{N}$. By Theorem 3.1, we have

$$
N^{-m} \int_{B\left(P_{N}, r_{N}\right)}\left|\Pi_{N}^{P_{N}}\right|^{2} d V \geq 1-o(1) .
$$


The same inequality holds for $Q_{N}$. If $\Phi_{N}\left(P_{N}\right)=\Phi_{N}\left(Q_{N}\right)$ then the total $\mathcal{L}^{2}$-norm of $\Pi_{N}(x, \cdot)$ would have to be $\sim 2 N^{m / 2}$, contradicting the asymptotic $\sim N^{m / 2}$ from Theorem 2(a).

To prove that case (ii) cannot occur, we assume on the contrary that $\Phi_{N}\left(P_{N}\right)=\Phi_{N}\left(Q_{N}\right)$, where $P_{N}=\rho_{N}(0)$ and $Q_{N}=\rho_{N}\left(\frac{v_{N}}{\sqrt{N}}\right), 0 \neq\left|v_{N}\right| \leq C$, using a Heisenberg coordinate chart $\rho_{N}$ about $P_{N}$. We consider the function

$$
f_{N}(t)=\frac{\left|\Pi_{N}^{P_{N}}\left(0, \frac{t v_{N}}{\sqrt{N}}\right)\right|^{2}}{\Pi_{N}^{P_{N}}(0,0) \Pi_{N}^{P_{N}}\left(\frac{t v_{N}}{\sqrt{N}}, \frac{t v_{N}}{\sqrt{N}}\right)} .
$$

Then $f_{N}(0)=1$ and we see from (85) and the Cauchy-Schwartz inequality that $f_{N} \leq 1$. Furthermore, since we are supposing that $\Phi_{N}\left(P_{N}\right)=\Phi_{N}\left(Q_{N}\right)$, we also have $f_{N}(1)=1$. Thus for some value of $t_{N}$ in the open interval $(0,1)$, we have $f_{N}^{\prime \prime}\left(t_{N}\right)=0$. By Theorem 3.1,

$$
f_{N}(t)=e^{-\left|v_{N}\right|^{2} t^{2}}\left[1+N^{-1 / 2} \widetilde{R}_{N}\left(t v_{N}\right)\right]
$$

where

$\widetilde{R}_{N}(v)=R_{1}\left(P_{N} ; 0, v, N\right)+R_{1}\left(P_{N} ; v, 0, N\right)-R_{1}\left(P_{N} ; v, v, N\right)-R_{1}\left(P_{N} ; 0,0, N\right)+O\left(N^{-1 / 2}\right)$.

The estimate for $R_{1}$ yields:

$$
\left\|\widetilde{R}_{N}\right\|_{\mathcal{C}^{2}\{|v| \leq C\}}=O(1)
$$

Since $f_{N}(1)=1$, it follows from (90)-(91) that $\left|v_{N}\right|^{2}=O\left(N^{-1 / 2}\right)$. (A more careful analysis shows that we can replace $N^{-1 / 2}$ with $N^{-1}$ in (90) and thus $\left|v_{N}\right|=O\left(N^{-1 / 2}\right)$.)

Write $e^{x}=1+x+x^{2} \varphi(x)$. We then have

$$
f_{N}(t)=1-\left|v_{N}\right|^{2} t^{2}+\left|v_{N}\right|^{4} t^{4} \varphi\left(\left|v_{N}\right|^{2} t^{2}\right)+N^{-1 / 2} \widetilde{R}_{N}\left(t v_{N}\right)\left[1-\left|v_{N}\right|^{2} t^{2}+\left|v_{N}\right|^{4} t^{4} \varphi\left(\left|v_{N}\right|^{2} t^{2}\right)\right] .
$$

Thus by (91),

$$
f_{N}^{\prime \prime}(t)=-2\left|v_{N}\right|^{2}+O\left(\left|v_{N}\right|^{4}\right)+O\left(N^{-1 / 2}\left|v_{N}\right|^{2}\right), \quad|t| \leq 1 .
$$

Since $\left|v_{N}\right|=o(1)$, it follows that

$$
0=f_{N}^{\prime \prime}\left(t_{N}\right)=(-2+o(1))\left|v_{N}\right|^{2}
$$

which contradicts the assumption that $v_{N} \neq 0$.

\section{Transversal SECTIONS}

5.1. Quantitative transversality. To illustrate the connection between our almost holomorphic sections and the asymptotically holomorphic sections of Donaldson and of Auroux, we now explain how to adapt Donaldson's proof of the existence of quantitatively transverse asymptotically holomorphic sections to our setting of almost holomorphic sections. We shall show that the normalized coherent states in our spaces $H_{J}^{0}\left(M, L^{N}\right)$ of almost holomorphic sections satisfy the estimates of Proposition 34 in Don1, suitably modified. Using these modified estimates together with the proof of Proposition 15 and of Lemma 14 in [Don1, one obtains the following result on the existence of almost holomorphic transversal sections.

Proposition 5.1. There is a constant $C<+\infty$ such that for all sufficiently large $N$, there exists a section $s_{N} \in H_{J}^{0}\left(M, L^{N}\right)$ with $\left\|\bar{\partial} s_{N}\right\|_{\infty} \leq C$ and which satisfies $\left|\partial s_{N}\right|>C^{-1} \sqrt{N}$ on $Z_{s_{N}}$. 
Here $Z_{s_{N}}$ denotes the zero set of the section $s_{N}$. The existence of asymptotically holomorphic sections satisfying these transversality estimates was given by Donaldson [Don1, Theorem 5]. (Donaldson expresses transversality in terms of the derivatives $\partial_{\text {scaled }}=\frac{1}{\sqrt{N}} \partial$, $\bar{\partial}_{\text {scaled }}=\frac{1}{\sqrt{N}} \bar{\partial}$ with respect to the scaled coordinates.) Recall that transversality implies that $Z_{s_{N}}$ is a symplectic submanifold of $M$ (whose homology class is the Poincare dual of $N[\omega]$ ).

Before we begin the proof of Proposition 5.1, we recall that the starting point of Donaldson's construction is the existence of concentrated asymptotically holomorphic sections $\sigma_{p}^{N}$ supported on a ball of radius $O\left(N^{-1 / 3}\right)$ about each point $p \in M$ satisfying certain $\mathcal{C}^{2}$ estimates [Don1, Proposition 11]. In the integrable case, Donaldson constructs holomorphic sections $\sigma_{p}^{N}$ satisfying the estimates (Don1, Proposition 34)

$$
\begin{array}{ll}
e^{-b d_{N}(p, q)^{2}} \leq\left|\sigma_{p}^{N}(q)\right| \leq e^{-a d_{N}(q, p)^{2}}, & d_{N}(q, p) \leq \epsilon N^{1 / 6} \\
\left|\sigma_{p}^{N}(q)\right| \leq e^{-a N^{1 / 3}}, & d_{N}(q, p) \geq \epsilon N^{1 / 6}
\end{array}
$$

where $d_{N}(q, p)=\sqrt{N} d(q, p)$ denotes the scaled distance on $M$. Given such sections, one forms the $\frac{1}{\sqrt{N}}$-'lattice' of points $\Lambda_{N}=\left\{p_{i}\right\}$ and the associated complex vector space of sections

$$
s_{N}=\sum_{i} w_{i} \sigma_{p_{i}}^{N}, \quad w_{i} \in \mathbb{C}
$$

Donaldson shows (Don1, Proposition 32) that one can choose the coefficients $\left\{w_{i}\right\}$ with $\left|w_{i}\right|<1$ so that the holomorphic section $s_{N}$ satisfies the quantitative transversality estimate

$$
\left|\partial s_{N}(z)\right|>C^{-1} \sqrt{N}, \quad \forall z \in Z_{s_{N}} .
$$

Our principal claim is that the Szegö kernel $\Pi_{N}(\bullet, p)$ itself, when normalized to have modulus one at $z=p$, satisfies estimates sufficient to prove the existence of transversal almost-holomorphic sections of the form (92). We define

$$
\sigma_{p}^{N}(x)=\frac{\Pi_{N}(x, p)}{\Pi_{N}(p, p)},
$$

so that $\sigma_{p}^{N}(p)=1$. (Recall that $\Pi_{N}(p, p)=\pi^{-m} N^{m}+O\left(N^{m-1}\right)$.)

Lemma 5.2. For all $\varepsilon, D \in \mathbb{R}^{+}$, we have for $N$ sufficiently large:

$$
\begin{array}{ll}
\text { (i) }\left|\sigma_{p}^{N}(q)\right| \geq\left[1-\frac{C}{\sqrt{N}} d_{N}(q, p)\right] e^{-\frac{1+\varepsilon}{2} d_{N}(q, p)^{2}}, \quad d_{N}(q, p) \leq D ; \\
\text { (ii) }\left|\sigma_{p}^{N}(q)\right| \lesssim\left[1+\frac{C}{\sqrt{N}} d_{N}(q, p)\right] e^{-\frac{1-\varepsilon}{2} d_{N}(q, p)^{2}}, \quad d_{N}(q, p) \leq N^{1 / 6} ; \\
\text { (iii) } \quad N^{-k / 2}\left|\nabla^{k} \sigma_{p}^{N}(q)\right| \lesssim C_{k} e^{-\frac{1-\varepsilon}{2} d_{N}(q, p)^{2}}, \quad k \geq 0 ; \\
\text { (iv) } \quad N^{-k / 2}\left|\nabla^{k} \bar{\partial} \sigma_{p}^{N}(q)\right| \lesssim C_{k} e^{-\frac{1-\varepsilon}{2} d_{N}(q, p)^{2}}, \quad k \geq 0 ;
\end{array}
$$

where $\alpha \lesssim \beta$ means that $\alpha-\beta \leq O\left(N^{-\infty}\right)$, uniformly for $(q, p) \in M \times M$. 
Since the length $\left|\sigma_{N}(p, \theta)\right|$ is independent of $\theta$, we have dropped $\theta$ from the notation and regard $\left|\sigma_{N}\right|$ as a function on $M$. Note that (iii) implies that

$$
\left|\nabla^{k} \sigma_{p}^{N}(q)\right|=O\left(N^{-\infty}\right), \quad \text { for } \quad \mathrm{d}_{\mathrm{N}}(\mathrm{q}, \mathrm{p}) \geq \mathrm{N}^{1 / 6}, \quad \mathrm{k}=0,1,2, \ldots .
$$

Proof. We let $q=p+\frac{u}{\sqrt{N}}$ (in preferred coordinates), so that $u \approx d_{N}(q, p)$, and we write $\sigma_{p}^{N}(q)=\sigma_{p}^{N}\left(\frac{u}{\sqrt{N}}, 0\right)$. The lower bound (i) is an immediate consequence of Theorem 3.1. To verify (ii)-(iv) for $u \leq N^{1 / 6}$ (i.e., on the balls of unscaled radii $N^{-1 / 3}$ ), we observe that the asymptotic expansion of Theorem 3.1 has the following extension to the scaled $N^{1 / 6}$ balls:

$$
\begin{aligned}
N^{-m} & \Pi_{N}^{P_{0}}\left(\frac{u}{\sqrt{N}}, \frac{\theta}{N} ; 0,0\right) \\
& =\Pi_{1}^{\mathbf{H}}(u, \theta ; 0,0)\left[1+\sum_{r=1}^{K} N^{-r / 2} b_{r}\left(P_{0}, u, 0\right)\right]+N^{-(K+1) / 2} R_{K}\left(P_{0}, u, N\right),
\end{aligned}
$$

where

$$
\left\|R_{K}\left(P_{0}, u, N\right)\right\|_{\mathcal{C}^{j}\left(\left\{|u| \leq N^{1 / 6}\right\}\right.} \lesssim C_{K, j} e^{-\frac{1-\varepsilon}{2}|u|^{2}} \quad \text { for } j \geq 0
$$

To verify (96), we modify the proof of Theorem 3.1 as follows: We first note that

$$
N R_{3}^{\psi}\left(\frac{u}{\sqrt{N}}, 0\right) \leq C, \quad \text { for }|u| \leq N^{1 / 6}
$$

and hence we obtain the large-ball version of (75):

$$
\begin{array}{r}
\left|A\left(t, \theta ; P_{0}, u, 0\right)\right| \leq C^{\prime} e^{-\Re g|u|^{2} / 2}\left|s\left(\frac{u}{\sqrt{N}}, \theta ; 0,0, N t\right)\right| \leq C^{\prime \prime} e^{-\frac{1-\varepsilon}{2}|u|^{2}} N^{m} \\
1-\delta \leq t \leq 3,-\delta \leq \theta \leq \delta,|u| \leq 2 N^{1 / 6}
\end{array}
$$

We use a smooth partition of unity to decompose the integral (70):

$$
I_{1}=I_{1}^{\prime}+I_{1}^{\prime \prime}, \quad I_{1}^{\prime}=N \int_{1-\delta}^{3} \int_{-\delta}^{\delta} e^{i N \Psi(t, \theta)} A\left(t, \theta ; P_{0}, u, 0\right) d \theta d t .
$$

The integral $I_{1}^{\prime \prime}$ is over a compact region outside the critical point $(1,0)$ of the phase $\Psi$. In fact $\left|\frac{\partial \Psi}{\partial \theta}\right|>\alpha>0$ in this region, and the method of proof that $I_{2}=O\left(N^{-\infty}\right)$ shows that we also have $I_{1}^{\prime \prime}=O\left(N^{-\infty}\right)$, uniformly for $|u| \leq N^{1 / 6}$ by (98).

Furthermore by (97), the asymptotic expansion (76) of the exponential $e^{N g R_{3}\left(\frac{u}{\sqrt{N}}, 0\right)}$ holds for $|u| \leq N^{1 / 6}$, and hence we have the large-ball version of $(78)$ :

$$
\begin{gathered}
A\left(t, \theta ; P_{0}, u, 0\right)=\rho_{1}(t) e^{-g|u|^{2} / 2} N^{m}\left[\sum_{n=0}^{K} N^{-n / 2} f_{n}\left(u, 0 ; t, \theta, P_{0}\right)+R_{K}(u, t, \theta)\right], \\
\left|R_{K}\right| \leq C_{K} O\left(N^{-\frac{K+1}{2}}\right) .
\end{gathered}
$$

The remainder estimate (96) now follows from (98) and (100) as in the proof of Theorem 3.1 .

To verify (ii) for $|u| \leq N^{1 / 6}$, we apply (96) (with $K=0$ ) to conclude that

$$
\left|\sigma_{p}^{N}\left(p+\frac{u}{\sqrt{N}}\right)\right|=e^{-\frac{1}{2}|u|^{2}}+\frac{1}{\sqrt{N}} e^{-\frac{1-\varepsilon}{2}|u|^{2}} R(p, u, N), \quad\left|D_{u}^{\alpha} R(p, u, N)\right| \leq C_{|\alpha|} .
$$

Since $\left|\sigma_{p}^{N}(p)\right|=1$, it follows that $R(p, 0, N)=0$ and hence

$$
R(p, u, N) \leq C|u|, \quad \text { for }|u|<N^{1 / 6} .
$$


Since $|u| \approx d_{N}\left(p, p+\frac{u}{\sqrt{N}}\right)$ for $|u|<\varepsilon \sqrt{N}$, the inequality (ii) follows from (101)-(102).

To show that (iii)-(iv) holds for $d_{N}(q, p) \leq N^{1 / 6}$, we lift a local frame $\left\{\bar{Z}_{j}^{M}\right\}$ of the form (匣) to obtain the local frame $\left\{\bar{Z}_{1}, \ldots, \bar{Z}_{m}\right\}$ for $H^{0,1} X$ given by

$$
\bar{Z}_{j}\left(p+\frac{u}{\sqrt{N}}\right)=\frac{\partial^{h}}{\partial \bar{z}_{j}}+\sum_{r=1}^{m} B_{j k}\left(\frac{u}{\sqrt{N}}\right) \frac{\partial^{h}}{\partial z_{k}}, \quad B_{j k}\left(\frac{u}{\sqrt{N}}\right)=O\left(\frac{1}{\sqrt{N}}|u|\right) \leq O\left(N^{-1 / 3}\right) .
$$

Recalling (6) and (14), we further have

$$
\frac{\partial^{h}}{\partial \bar{z}_{j}}=\frac{\partial}{\partial \bar{z}_{j}}+\left[-\frac{i}{2} \frac{u_{j}}{\sqrt{N}}-R_{1}^{\bar{A}_{j}}\left(\frac{u}{\sqrt{N}}\right)\right] \frac{\partial}{\partial \theta}=\frac{\partial}{\partial \bar{z}_{j}}+i \sqrt{N} u_{j}-i N R_{1}^{\bar{A}_{j}}\left(\frac{u}{\sqrt{N}}\right) \text { at } p+\frac{u}{\sqrt{N}}
$$

where $R_{1}^{\bar{A}_{j}}\left(\frac{u}{\sqrt{N}}\right)=O\left(\frac{1}{N}|u|^{2}\right)$. The inequalities (iii)-(iv) for $|u| \leq N^{1 / 6}$ follow as before, using (103)-(104).

It remains to show (95). For this it suffices to show that $\nabla^{k} \Pi_{N}^{p}\left(\frac{u}{\sqrt{N}}, 0 ; 0,0\right)=O\left(N^{-\infty}\right)$ for $|u| \geq N^{1 / 6}$. Since $\Pi_{N}=S_{N}+E_{N}$, where $E_{N}(x, y)=O\left(N^{-\infty}\right)$, we can neglect the remainder $E_{N}$. Furthermore, since $S_{N}$ is rapidly decaying away from the diagonal, it suffices to consider

$$
N^{1 / 6} \leq|u| \leq \varepsilon N^{1 / 2}
$$

As in the proof of Theorem 3.1, we use a smooth partition of unity to write (60) as the sum of two integrals $I_{1}, I_{2}$. Note that in the proof that $I_{2}=O\left(N^{-\infty}\right)$, we use only that $|u| \leq \varepsilon N^{1 / 2}$. So it remains to consider the integral $I_{1}$. Recalling (65), we have

$$
\widetilde{\Psi}=\alpha_{N}(\theta) t-\theta, \quad\left|\alpha_{N}(\theta)\right| \geq \frac{|u|^{2}}{3 N} \geq \frac{1}{3} N^{-2 / 3} \quad \text { for } \quad N^{1 / 6} \leq|u| \leq \varepsilon N^{1 / 2} .
$$

Integrating by parts, we then obtain

$$
\begin{aligned}
& \left|\int_{0}^{3} e^{i N \widetilde{\Psi}} \rho_{1}(t) s\left(\frac{u}{\sqrt{N}}, \theta ; \frac{v}{\sqrt{N}}, 0, N t\right) d t\right| \\
& \quad \leq\left|N \alpha_{N}\right|^{-k} \sup _{t \leq 3}\left|\frac{\partial^{k}}{\partial t^{k}}\left[\rho_{1}(t) s\left(\frac{u}{\sqrt{N}}, \theta ; \frac{v}{\sqrt{N}}, 0, N t\right)\right]\right| \leq C_{k} N^{-k / 3+m},
\end{aligned}
$$

Hence by $(66)$,

$$
\left|I_{1}\right| \leq C_{k}^{\prime} N^{-k / 3+m+1}
$$

and thus $S_{N}^{p}\left(\frac{u}{\sqrt{N}}, 0 ; 0,0\right)=O\left(N^{-\infty}\right)$. The estimate for the derivatives is similar.

Remark: It is of some interest to know how precise these off-diagonal estimates actually are. In the holomorphic case (with a $\mathcal{C}^{\infty}$ but not necessarily analytic metric), M. Christ has shown (cf. [Chr) that one has the global off-diagonal bounds

$$
\left|\Pi_{N}(x, y)\right| \leq C N^{m} e^{-\sqrt{N} d(x, y)}, \quad((x, y) \in X \times X)
$$

for a constant $C$ which is uniform in $(N, x, y)$. He also shows that this estimate cannot be improved in general. Thus, the decay estimates of Lemma 5.2 do not hold in general without the $O\left(N^{-\infty}\right)$ term. It is quite possible that (106) extends to the almost complex context of this paper, since it is based on weighted $\mathcal{L}^{2}$ estimates for an associated Green's kernel which seem to have natural analogues for the pseudodifferential $\bar{D}_{j}$-complex of $B$ Band. However, we do not need such a precise estimate as (106) and hence will not investigate it further. 
We now outline how Proposition 5.1 follows from Lemma 5.2. The main steps in adapting the proof of Donaldson's transversality theorem [Don1, Theorem 5] are to show the following: Let

then

$$
\mathcal{G}_{p_{i}}^{N}(q)=\left|\sigma_{p_{i}}^{N}(q)+\right| \bar{\partial} \sigma_{p_{i}}^{N}(q)\left|+\frac{1}{\sqrt{N}}\right| \nabla \sigma_{p_{i}}^{N}(q)\left|+\frac{1}{\sqrt{N}}\right| \nabla \bar{\partial} \sigma_{p_{i}}^{N}(q)\left|+\frac{1}{N}\right| \nabla^{2} \sigma_{p_{i}}^{N}(q) \mid
$$

- $\sum_{i} \mathcal{G}_{p_{i}}^{N}(q)$ is bounded independently of $N$;

- for all $D>0$ there exists $N_{0}(D)$ such that $\mathcal{G}_{p_{i}}^{N}(q) \leq C e^{-D^{2} / 3}$ for $d_{N}\left(q, p_{i}\right) \geq D$, $N>N_{0}(D)$.

These statements follow immediately from parts (iii) and (iv) of Lemma 5.2; to obtain the first statement we also note that the number of points of the 'lattice' $\Lambda_{N}=\left\{p_{i}\right\}$ is $\sim N^{m}$ and that we can choose $D>0$ so that the balls $\left\{B\left(p_{i}, \frac{D}{\sqrt{N}}\right)\right\}$ cover $M$ and at most $(2 D+2)^{2 m}$ of them pass through any point. From there (as in the holomorphic case described in [Don1, p. 697]), we obtain Proposition 5.1 from the argument in [Don1, $\S \S 3-4]$.

5.2. The Adjunction Formula. As explained in [Don1], the zero set $\mathbf{Z}\left(s_{N}\right)$ of a section $s_{N} \in \mathcal{C}^{\infty}\left(M, L^{N}\right)$ satisfying the transversality condition (93) is a symplectically embedded submanifold. In particular, when $(M, \omega)$ is a 4 -manifold and $\mathbf{Z}\left(s_{N}\right)$ is a symplectically embedded Riemann surface, then it is well known that the genus $g_{N}$ of $\mathbf{Z}\left(s_{N}\right)$ is given by the Adjunction Formula as in the holomorphic case:

$$
g_{N}=\frac{c_{1}(L)^{2}}{2} N^{2}-\frac{c_{1}(M) \cdot c_{1}(L)}{2} N+1 .
$$

To verify (107), we let $C$ denote the complex curve $\mathbf{Z}\left(s_{N}\right)$ with complex structure $J_{0}$ induced by $\omega$. Then $J_{0}$ can be extended to an almost complex structure $J^{\prime}$ on $M$, compatible with $\omega$, and it follows that $C$ is a J-holomorphic curve in $\left(M, \omega, J^{\prime}\right)$. By our assumptions, the homomorphism $\nabla s_{N}: T_{M} \rightarrow L^{N}$ is surjective along $C$. Hence, for all $z_{0} \in C$, we have

$$
\operatorname{ker}_{z_{0}}\left(\partial s_{N}: T_{M}^{1,0} \rightarrow L_{N}\right)=\left(\operatorname{ker}_{z_{0}} \nabla s_{N}\right) \cap T_{M, z_{0}}^{1,0}=T_{C, z_{0}} \cap T_{M, z_{0}}^{1,0}=T_{C, z_{0}}^{1,0}
$$

(where we use the almost complex structures $J^{\prime}, J_{0}$ on $M, C$, respectively). Proceeding as in the holomorphic case, from the resulting exact sequence of vector bundles

$$
\left.\left.0 \rightarrow T_{C}^{1,0} \rightarrow T_{M}^{1,0}\right|_{C} \stackrel{\partial s_{N}}{\rightarrow} L^{N}\right|_{C} \rightarrow 0,
$$

we obtain the Adjunction Formula:

$$
\left.\left(K_{M} \otimes L^{N}\right)\right|_{C}=K_{C}
$$

Here $K_{M}=\bigwedge^{2} T_{M}^{1,0^{*}}$ and $K_{C}=T_{C}^{1,0 *}$. Note that $c_{1}\left(K_{M}\right)$ is independent of the choice of $J^{\prime}$, since the space of compatible almost complex structures on $(M, \omega)$ is connected (and in fact is contractible; see McSa, 4.1]). Thus we have $c_{1}\left(K_{M}\right)=-c_{1}(M)$. It follows further from Donaldson's Lefschetz hyperplane theorem for asymptotically holomorphic sections satisfying (93) (Don1, Prop. 39]) that $C$ is connected. We then conclude from (108) that

$$
2 g-2=c_{1}\left(K_{C}\right)=\left(-c_{1}(M)+N c_{1}(L)\right) \cdot N c_{1}(L),
$$

which yields (107).

Auroux Aur1] generalized Donaldson's construction to obtain asymptotically holomorphic, 'quantitatively transversal' sections of $E \otimes L^{N}$, where $\mathrm{E}$ is an arbitrary complex vector 
bundle of rank $k \leq m$ over $M$. A section $s_{N} \in \mathcal{C}^{\infty}\left(M, E \otimes L^{N}\right)$ is quantitatively transversal (Aur1, Definition 2) if $\nabla s_{N}: T_{M} \rightarrow E \otimes L^{N}$ is surjective and has a right inverse of norm $<\frac{1}{\eta \sqrt{N}}$ at all points where $\left|s_{N}\right|<\eta$. The zero sets $\mathbf{Z}\left(s_{N}\right)$ are then embedded symplectic submanifolds of $M$ (Aur1, Prop. 1). In particular, for the case where $E$ is the trivial vector bundle $M \times \mathbb{C}^{k}$, sections of $E \otimes L^{N}$ are $k$-tuples of sections of $L^{N}$, and we can write $s_{N}=\left(s_{N}^{1}, \ldots, s_{N}^{k}\right)$. Then $\mathbf{Z}\left(s_{N}\right)$ is the set of simultaneous zeros of the sections $s_{N}^{j} \in H_{J}^{0}\left(M, L^{N}\right)$ and $s_{N}$ is asymptotically holomorphic if the $s_{N}^{j}$ are asymptotically holomorphic (but quantitative transversality of the $s_{N}^{j}$ is not sufficient to guarantee transversality of $\left.s_{N}\right)$.

By the previous comments, Auroux's argument can be used to give asymptotically holomorphic, quantitatively transverse sections $s_{N} \in H_{J}^{0}\left(M, L^{N}\right)^{k}$. In the case where $k=m-1$, $\mathbf{Z}\left(s_{N}\right)$ is a complex curve, which is connected by Auroux's generalization [Aur1, Prop. 2] of Donaldson's Lefschetz hyperplane section theorem cited above, and the genus formula (107) extends to this case as follows:

Proposition 5.3. (see Aur1, Proposition 5]) Let $s_{N}^{1}, \ldots, s_{N}^{m-1} \in \mathcal{C}^{\infty}\left(M, L^{N}\right)$ such that $s_{N}:=\left(s_{N}^{1}, \ldots, s_{N}^{m-1}\right)$ is quantitatively transverse and asymptotically holomorphic, for $N=$ $1,2,3, \ldots$. Then for $N$ sufficiently large, $\mathbf{Z}\left(s_{N}\right)$ is connected and its genus $g_{N}$ is given by

$$
g_{N}=\frac{m-1}{2} c_{1}(L)^{m} N^{m}-\frac{1}{2} c_{1}(M) \cdot c_{1}(L)^{m-1} N^{m-1}+1 .
$$

The proposition follows by the argument in Aur1, which is a generalization of the proof of (107). To summarize this argument, the hypotheses imply that $\mathbf{Z}\left(s_{N}\right)$ is a symplectic submanifold (for $N \gg 0$ ) and one obtains as before an exact sequence

$$
\left.\left.0 \rightarrow T_{C}^{1,0} \rightarrow T_{M}^{1,0}\right|_{C} \stackrel{\partial s_{N}}{\longrightarrow} L^{N} \oplus \cdots \oplus L^{N}\right|_{C} \rightarrow 0
$$

which yields

$$
\begin{aligned}
2-2 g_{N} & =c_{1}(C)=\left(c_{1}(M)-(m-1) N c_{1}(L),[C]\right) \\
& =\left[c_{1}(M)-(m-1) N c_{1}(L)\right] \cdot c_{1}(L)^{m-1} N^{m-1} .
\end{aligned}
$$

Remark: The results of this paper can be generalized to the case of almost holomorphic sections in $H_{J}^{0}\left(M, E \otimes L^{N}\right)$, for an arbitrary complex vector bundle $E \rightarrow M$. Auroux's argument can then be used to find quantitatively transversal, asymptotically holomorphic sections $s_{N} \in H_{J}^{0}\left(M, E \otimes L^{N}\right)$. The zero sets $\mathbf{Z}\left(s_{N}\right)$ are then $(m-k-1)$-connected symplectic submanifolds, for $N \gg 0$; if $k=\operatorname{rank} E=m-1$, they are connected Riemann surfaces. In this case, the genus formula becomes:

$$
2 g_{N}-2=\left[(m-1) N c_{1}(L)+c_{1}(E)-c_{1}(M)\right] \sum_{j=0}^{m-1} c_{m-1-j}(E) c_{1}(L)^{j} N^{j} .
$$

To verify (110), we proceed as in Aur1, noting first that we have an exact sequence

$$
\left.\left.0 \rightarrow T_{C}^{1,0} \rightarrow T_{M}^{1,0}\right|_{C} \stackrel{\partial s_{N}}{\longrightarrow} E \otimes L^{N}\right|_{C} \rightarrow 0 .
$$

The genus formula (119) then follows from (111) by a routine Chern class calculation. 


\section{REFERENCES}

[Aur1] D. Auroux, Asymptotically holomorphic families of symplectic submanifolds. Geom. Funct. Anal. 7 (1997), 971-995.

[Aur2] D. Auroux, Symplectic 4-manifolds as branched coverings of $\mathbb{C P}^{2}$, Invent. Math. 139 (2000), $551-602$. .

[AuKa] D. Auroux and L. Katzarkov, Branched coverings of $\mathbb{C P}^{2}$ and invariants of symplectic 4-manifolds (preprint, 1999).

[BSZ1] P. Bleher, B. Shiffman and S. Zelditch, Universality and scaling of correlations between zeros on complex manifolds, Invent. Math., 142 (2000), 351-395.

[BSZ2] P. Bleher, B. Shiffman and S. Zelditch, Universality and scaling of zeros on symplectic manifolds, to appear in MSRI volume on Random Matrices.

[BoUr1] D. Borthwick and A. Uribe, Almost complex structures and geometric quantization, Math. Res. Lett. 3 (1996), 845-861.

[BoUr2] D. Borthwick and A. Uribe, Nearly Kählerian embeddings of symplectic manifolds, Asian J. Math. 4 (2000), 599-620.

[Bch] T. Bouche, Asymptotic results for Hermitian line bundles over complex manifolds: the heat kernel approach. Higher-dimensional complex varieties (Trento, 1994), 67-81, de Gruyter, Berlin, 1996.

[Bout] L. Boutet de Monvel, Hypoelliptic operators with double characteristics and related pseudodifferential operators, Comm. Pure and Appl. Math. 27 (1974), 585-639.

[BoGu] L. Boutet de Monvel and V. Guillemin, The Spectral Theory of Toeplitz Operators, Ann. Math. Studies 99, Princeton Univ. Press, Princeton, 1981.

[BoSj] L. Boutet de Monvel and J. Sjöstrand, Sur la singularité des noyaux de Bergman et de Szegö, Asterisque 34-35 (1976), 123-164.

[Cat] D. Catlin, The Bergman kernel and a theorem of Tian, in: Analysis and Geometry in Several Complex Variables, G. Komatsu and M. Kuranishi, eds., Birkhäuser, Boston, 1999.

[Chr] M. Christ, On the $\bar{\partial}$ equation in weighted $L^{2}$ norms in $\mathbb{C}^{1}$, Jour. Geom. Anal. 3 (1991), 193-230.

[Don1] S. Donaldson, Symplectic submanifolds and almost complex geometry, J. Diff. Geom. 44 (1996), 666-705.

[Don2] S. Donaldson, Lefschetz fibrations in symplectic geometry, Proceedings of the International Congress of Mathematicians, Vol. II (Berlin, 1998).

[GuSt] V. Guillemin and S. Sternberg, Symplectic Techniques in Physics, Cambridge Univ. Press, Cambridge, 1984.

[GuUr] V. Guillemin and A. Uribe, Laplace operator on tensor powers of a line bundle, Asympt. Anal. 1 (1988), 105-113.

[Hör] L. Hörmander, The Analysis of Linear Partial Differential Operators, I, II, III, IV, Springer Verlag, N.Y., 1983, 1983, 1985, 1994.

[McSa] D. McDuff and D. Salamon, Introduction to Symplectic Topology, Oxford Univ. Press, Oxford, 1995.

[MelSj] A. Melin and J. Sjöstrand, Fourier integral operators with complex valued phase functions, Springer Lecture Notes 459, pp. 120-233, Springer, N.Y., 1975.

[MenSj] A. Menikoff and J. Sjöstrand, On the eigenvalues of a class of hypoelliptic operators, Math. Ann. 235 (1978), 55-85.

[ShZe1] B. Shiffman and S. Zelditch, Distribution of zeros of random and quantum chaotic sections of positive line bundles, Comm. Math. Phys., 200 (1999), 661-683.

[ShZe2] B. Shiffman and S. Zelditch, Random polynomials of high degree and Levy's concentration of measure, in preparation.

[Sik] J. C. Sikorav, Construction de sous-variétés symplectiques (d'après S. K. Donaldson et D. Auroux), Séminaire Bourbaki. Vol. 1997/98. Astérisque 252, (1998), 231-253.

[Sjö] J. Sjöstrand, Density of resonances for strictly convex analytic obstacles, Can. J. Math. 48 (1996), 397-447.

[Tian] G. Tian, On a set of polarized Kähler metrics on algebraic manifolds, J. Diff. Geometry 32 (1990), 99-130. 
[Woo] N. M. J. Woodhouse, Geometric Quantization, Clarendon Press, Oxford, 1992.

[Zel] S. Zelditch, Szegö kernels and a theorem of Tian, Int. Math. Res. Notices 6 (1998), 317-331.

Department of Mathematics, Johns Hopkins University, Baltimore, MD 21218, USA

E-mail address: shiffman@math.jhu.edu, zelditch@math.jhu.edu 